Optimal two-level conjoint designs for large numbers of attributes

Roselinde Kessels, Peter Goos and Martina Vandebroek

DEPARTMENT OF DECISION SCIENCES AND INFORMATION MANAGEMENT (KBI) 


\title{
Optimal two-level conjoint designs for large numbers of attributes
}

\author{
Roselinde Kessels* \\ Peter Goos ${ }^{\dagger}$ \\ Martina Vandebroek ${ }^{\ddagger}$
}

\begin{abstract}
In this paper, we propose a simple strategy to construct $\mathcal{D}-, \mathcal{A}-, \mathcal{G}^{-}$and $\mathcal{V}$-optimal two-level multi-attribute designs for rating-based conjoint studies. Our approach combines orthogonal designs and balanced or partially balanced incomplete block designs. In order not to overload respondents with complicated tasks, the designs hold one or more attributes at a constant level. The designs are variance-balanced meaning that they yield an equal amount of information on each of the part-worths. Some examples are provided to illustrate the method.
\end{abstract}

Keywords: two-level conjoint designs, $\mathcal{D}$-, $\mathcal{A}$-, $\mathcal{G}$ - and $\mathcal{V}$-optimality, orthogonal designs, balanced and partially balanced incomplete block designs

\section{Introduction}

A conjoint experiment tries to elucidate consumer preferences for the attributes of a good, that is a product or a service. This is usually done by asking respondents to rate a set of profiles or alternatives of the good. A profile involves a combination of levels of a set of predefined attributes of the good. Some of the administered profiles describe existing goods, whereas others describe hypothetical and possibly prospective goods. The objective of a conjoint experiment is to elicit as much information as possible on the utilities people derive from the attribute levels. These utilities are also called part-worths and correspond to the parameters of a statistical model. By means of accurate parameter estimates, precise predictions are aimed to depict consumers' purchasing behavior in a given market. Companies can then develop new goods that lead to a substantial rise in clientele.

${ }^{*}$ Department of Decision Sciences and Information Management, Faculty of Economics and Applied Economics, Katholieke Universiteit Leuven, Naamsestraat 69, 3000 Leuven, Belgium.

${ }^{\dagger}$ Department of Mathematics, Statistics and Actuarial Sciences, Faculty of Applied Economics, Universiteit Antwerpen, Prinsstraat 13, 2000 Antwerpen, Belgium.

${ }^{\ddagger}$ Department of Decision Sciences and Information Management \& University Center for Statistics, Katholieke Universiteit Leuven, 3000 Leuven, Belgium. 
To obtain precise parameter estimates, an efficient conjoint design needs to be constructed. The conjoint designs we set up in this paper are intended for screening out the vital few important attributes from a group of many potential ones. The different attributes involved in the conjoint designs have two levels each. In most conjoint studies, see e.g. Danaher (1997), Pullman et al. (2002) and references therein, the conjoint design consists of blocks or sets of an equal number of profiles. Each of these sets is evaluated by a different respondent. To be able to estimate all part-worths, each respondent is administered a different set of profiles.

The incorporation of large numbers of attributes in the design of conjoint experiments requires some special attention. As Green (1974) and Schwabe et al. (2003) have argued, respondents may get overloaded when they have to assimilate profiles that embrace more than four different attribute levels. Therefore, to overcome respondent fatigue, we only vary the levels of maximum four attributes in each of the profile sets assigned to the respondents. The profiles are still combinations of levels for all attributes, but the levels of one or more attributes do not vary between the profiles in a set. Each respondent thus rates a set of profiles in which the levels of one or more attributes are held constant. These constant attributes need not necessarily be the same in each profile set. To keep the profile sets comprehensible for the respondents, we confine ourselves to a maximum of ten constant attributes in the design.

Because each of the profile sets has a number of attributes at a constant level, the conjoint designs addressed in this paper show some similarities to split-plot designs. Split-plot designs are heavily used in industry when the levels of some of the experimental factors are difficult or costly to change or control. These factors are called whole plot factors and are kept at a constant level for several observations in the design. The other factors whose levels may vary are called sub-plot factors. Analogous to our type of conjoint design is that a split-plot design consists of blocks or groups of runs with the whole plot factors acting at a constant level. These blocks are termed whole plots. A split-plot design differs from our conjoint design in that the whole plot factors are naturally the same in each whole plot, whereas the constant attributes in a conjoint design may vary from set to set. We refer to the work of Goos and Vandebroek (2001; 2004) and Goos (2002; 2006a) for more details on split-plot designs.

Our approach to construct multi-attribute conjoint designs exploits the same linear model that is used for generating split-plot designs. In the split-plot setting, the model includes a random effect representing the whole plot variation. In our conjoint setting, the random effect is attributable to a respondent who rates a set of profiles. The reason is that respondents are assumed to be heterogeneous, meaning that profile ratings from the same respondent are more similar than profile ratings from different respondents. A random effect for each respondent accommodates this heterogeneity. Respondents are thereby supposed to be randomly selected from a prespecified market population. In the conjoint setting, we refer to the model as the linear random respondent effects model. Also Brazier et al. (2002) and Kessels et al. (2004) adopted this model for conjoint applications. Because the conjoint designs in this paper are set up for detecting the significant 
attributes from a large number of attributes, only main-effects models are considered.

Our design construction approach is conceptually easy to understand and generates designs with sets of 2, 4 and 8 profiles. In principle, our approach can produce designs with profile sets of sizes equal to a power of two. However, we restrict ourselves to a maximum size of eight as respondents can easily become fatigued by having to evaluate more than eight profiles. Related to conjoint designs with sets of two profiles are paired comparison designs (Grossmann et al. 2002 \& 2005; Grasshoff et al. 2004; Street et al. 2001; Street and Burgess 2004). These designs also comprise sets of two profiles, but in contrast with conjoint designs, each respondent evaluates all the sets. This is done by specifying the preferred profile in each set, and possibly also the preference strength.

Street et al. (2001) demonstrated that holding the levels of one or more attributes constant in two-level paired comparison designs leads to information losses when maineffects models are considered. This result also applies to conjoint designs. Keeping the rating tasks manageable for the respondents thus comes at a loss of information on the part-worths. Since all part-worths are assumed to be on the same footing, we look for conjoint designs that spread the information losses evenly over each part-worth. In other words, we want to set up conjoint designs that provide an equal amount of information on each part-worth. These conjoint designs are called variance-balanced conjoint designs.

To allow for variance balance in the conjoint designs, the constant attributes have to differ between the profile sets in such a way that each attribute is constant in an equal number of sets. In case of more than one constant attribute, we obtain an appropriate pattern of constant attributes using a balanced or partially balanced incomplete block design (BIBD or PBIBD). The levels in these one-factor block designs, called treatments, each occur the same number of times, which paves the way for the production of variancebalanced conjoint designs. To ensure the optimality of the designs, meaning that they yield maximum information on each part-worth, we choose the design profiles so that they form an orthogonal array. These designs offer the advantage that parameter estimates are statistically independent of each other.

The combination of orthogonal designs and BIBDs or PBIBDs has also been employed by Green (1974) to develop multi-attribute choice experiments for an equal and unequal number of levels for the attributes. Yet, the resulting designs are not guaranteed to be optimal as Grossmann et al. (2002) have shown. In choice experiments, respondents are administered a series of choice sets that each comprise several profiles. The respondents then indicate their preferred profile in every choice set. The underlying model is usually nonlinear, which requires the specification of prior parameter values before deriving the design. However, Green (1974) assumed zero prior values, hereby simplifying the nonlinear design problem to a linear one. Because of this assumption, the designs generated can also be utilized for conjoint experiments.

In one of his examples, Green (1974) constructed designs for partial profiles. These profiles are described by only a subset of the attributes. The levels of the other attributes 
are left unspecified. A BIBD is used to determine which of the attributes constitute the profiles. An orthogonal design is then assigned to each combination of attributes selected by the BIBD to form the profiles for these attributes. This approach yields variancebalanced designs when the attributes have two levels each. It best resembles our strategy since the attributes provided by the BIBD are the non-constant attributes in our conjoint setting. However, we also determine the levels of the other attributes, the constant attributes in our setting, so as to construct conjoint designs for full profiles. Comparing ratings from different profiles is impossible otherwise.

Based on a different approach, Street and Burgess (2004) generated optimal two-level paired comparison designs that can also be employed as variance-balanced conjoint designs for sets of two profiles. In this case, the paired comparison designs are set up for full profiles in which the levels of one or more attributes are held constant. They are referred to as constant difference pairs. Street and Burgess (2004) propose the use of generators and orthogonal designs to construct the pairs. They exploited the nonlinear Bradley-Terry model for choice experiments. Because zero prior parameter values are assumed, the model comes down to the linear model. The construction of variance-balanced constant difference pairs is illustrated in an empirical study by Severin (2000).

The outline of the remainder of the paper is as follows. Section 2 reviews the random respondent effects model used in conjoint design. In Section 3 we discuss the optimality conditions and in Section 4 we refine these conditions to deal with large numbers of attributes. We explain our design construction approach in Section 5 and describe the information content of the resulting designs in Section 6. Section 7 concludes the paper and highlights some further research possibilities.

\section{The random respondent effects model}

The model used to set up and analyze two-level multi-attribute conjoint experiments is the random respondent effects model. In this model, it is assumed that respondents are heterogeneous and randomly selected from a prespecified population. Each respondent $i, i=1, \ldots, b$, rates a different block or set of profiles to estimate all parameters. For convenience, the profile sets assigned to the $b$ respondents have the same size $m$. As a result, the total number of experimental profiles amounts to $n=b m$. Our approach to properly design two-level conjoint studies considers the cases where $m$ equals either 2 , 4 or 8 . Subsequently, the rating $U_{i j}$ for profile $j, j=1, \ldots, m$, by respondent $i$ is modelled as

$$
U_{i j}=\mathbf{x}_{i j}^{\prime} \boldsymbol{\beta}+\gamma_{i}+\varepsilon_{i j}
$$

In the model, $\mathbf{x}_{i j}$ is a $(k+1) \times 1$ vector with a one as first element and the attribute levels describing profile $j$ that is rated by respondent $i$ as the remaining $k$ elements. The attributes are quantitative or categorical factors that have two levels each. One level is coded as -1 and the other level as +1 . Consequently, each of the individual attribute levels occupies one entry in the vector $\mathbf{x}_{i j}$. Moreover, only main-effects models are consid- 
ered so that the number of attributes involved in the design amounts to $k$. $\boldsymbol{\beta}=\left[\beta_{0}, \ldots, \beta_{k}\right]^{\prime}$ is the $(k+1) \times 1$ unknown parameter vector with $\beta_{0}$ the intercept and $\beta_{1}, \ldots, \beta_{k}$ the partworths or weights attached to the attribute levels. $\gamma_{i}$ represents the random effect of respondent $i$ and $\varepsilon_{i j}$ is a random error term.

In matrix notation, model (1) becomes

$$
\boldsymbol{U}=\mathbf{X} \boldsymbol{\beta}+\mathbf{Z} \gamma+\varepsilon,
$$

where $\boldsymbol{U}$ is a vector of $n$ profile ratings, the vector $\gamma=\left[\gamma_{1}, \ldots, \gamma_{b}\right]^{\prime}$ contains the $b$ random respondent effects and $\boldsymbol{\varepsilon}$ is a random error vector. The matrices $\mathbf{X}$ and $\mathbf{Z}$ have dimensions $n \times(k+1)$ and $n \times b$, respectively. $\mathbf{X}$ is given by $\left[\mathbf{X}_{1}^{\prime}, \ldots, \mathbf{X}_{b}^{\prime}\right]^{\prime}$, where $\mathbf{X}_{i}=\left[\mathbf{x}_{i 1}, \ldots, \mathbf{x}_{i m}\right]^{\prime}$ collects the $m$ profiles rated by respondent $i . \mathbf{Z}=\mathbf{I}_{b} \otimes \mathbf{1}_{m}$, where $\otimes$ is the Kronecker product and $\mathbf{1}_{m}$ an $m \times 1$ vector of ones. It is assumed that

$$
\begin{gathered}
\mathrm{E}(\boldsymbol{\varepsilon})=\mathbf{0}_{n} \text { and } \operatorname{Cov}(\boldsymbol{\varepsilon})=\sigma_{\boldsymbol{\varepsilon}}^{2} \mathbf{I}_{n}, \\
\mathrm{E}(\boldsymbol{\gamma})=\mathbf{0}_{b} \text { and } \operatorname{Cov}(\boldsymbol{\gamma})=\sigma_{\boldsymbol{\gamma}}^{2} \mathbf{I}_{b}, \\
\text { and } \operatorname{Cov}(\boldsymbol{\gamma}, \boldsymbol{\varepsilon})=\mathbf{0}_{b \times n},
\end{gathered}
$$

where $\sigma_{\varepsilon}^{2}$ is the variance within respondents and $\sigma_{\boldsymbol{\gamma}}^{2}$ is the variance between respondents. Under these assumptions, the variance-covariance matrix $\mathbf{V}$ of the profile ratings $\boldsymbol{U}$ is $\mathbf{I}_{b} \otimes \mathbf{V}_{m}$, with

$$
\mathbf{V}_{m}=\sigma_{\varepsilon}^{2} \mathbf{I}_{m}+\sigma_{\gamma}^{2} \mathbf{1}_{m} \mathbf{1}_{m}^{\prime}=\sigma_{\boldsymbol{\varepsilon}}^{2}\left(\mathbf{I}_{m}+\frac{\rho}{1-\rho} \mathbf{1}_{m} \mathbf{1}_{m}^{\prime}\right)
$$

In this expression, $\rho=\sigma_{\gamma}^{2} /\left(\sigma_{\varepsilon}^{2}+\sigma_{\gamma}^{2}\right)$. This ratio measures the proportion of the total variance that is accounted for by the differences between respondents. It represents the degree of correlation between the ratings from a single respondent.

The vector of the unknown fixed model parameters $\boldsymbol{\beta}$ can be estimated using the generalized least squares estimator

$$
\hat{\boldsymbol{\beta}}=\left(\mathbf{X}^{\prime} \mathbf{V}^{-1} \mathbf{X}\right)^{-1} \mathbf{X}^{\prime} \mathbf{V}^{-1} \boldsymbol{U},
$$

with $\left(\mathbf{X}^{\prime} \mathbf{V}^{-1} \mathbf{X}\right)^{-1}$ the variance-covariance matrix of $\hat{\boldsymbol{\beta}}$, the inverse of which is the information matrix on $\boldsymbol{\beta}$.

\section{Design optimality}

Our strategy to construct efficient multi-attribute conjoint designs is based on the information matrix $\mathbf{X}^{\prime} \mathbf{V}^{-1} \mathbf{X}$ for the random respondent effects model (2). Goos and Vandebroek (2001) showed that the information matrix of a design $\mathbf{X}$ with profile sets of size $m$ can be written as

$$
\mathbf{X}^{\prime} \mathbf{V}^{-1} \mathbf{X}=\sigma_{\varepsilon}^{-2}\left\{\mathbf{X}^{\prime} \mathbf{X}-\frac{\rho}{1+\rho(m-1)} \sum_{i=1}^{b}\left(\mathbf{X}_{i}^{\prime} \mathbf{1}_{m}\right)\left(\mathbf{X}_{i}^{\prime} \mathbf{1}_{m}\right)^{\prime}\right\}
$$


For notational ease, we define the matrices $\mathbf{A}$ and $\mathbf{B}$ as

$$
\mathbf{A}=\mathbf{X}^{\prime} \mathbf{X} \text { and } \mathbf{B}=\sum_{i=1}^{b}\left(\mathbf{X}_{i}^{\prime} \mathbf{1}_{m}\right)\left(\mathbf{X}_{i}^{\prime} \mathbf{1}_{m}\right)^{\prime},
$$

so that the information matrix $\mathbf{X}^{\prime} \mathbf{V}^{-1} \mathbf{X}$ is of the form $\mathbf{A}-f(\rho) \mathbf{B}$.

The designs we derive are optimal with regard to four criteria that are functions of the information matrix (8) irrespective of the value of $\rho$. These criteria are the $\mathcal{D}_{-}, \mathcal{A}-$, $\mathcal{G}$ - and $\mathcal{V}$-optimality criteria. The $\mathcal{D}$ - and $\mathcal{A}$-optimality criteria both are concerned with a precise estimation of the parameters $\boldsymbol{\beta}$ in model $(2)$. A $\mathcal{D}$-optimal design minimizes the determinant of the variance-covariance matrix $\left(\mathbf{X}^{\prime} \mathbf{V}^{-1} \mathbf{X}\right)^{-1}$ or, equivalently, maximizes the determinant of the information matrix (8). An $\mathcal{A}$-optimal design minimizes the trace of the variance-covariance matrix. The $\mathcal{G}$ - and $\mathcal{V}$-optimality criteria are concerned with making precise response predictions. Because conjoint experiments particularly focus on producing precise predictions, these criteria are vital in design construction. The $\mathcal{G}$-optimality criterion seeks designs that minimize the maximum prediction variance over the region of interest, whereas the $\mathcal{V}$-optimality criterion seeks designs that minimize the average prediction variance over the region of interest.

To obtain conjoint designs that are $\mathcal{D}$-, $\mathcal{A}$-, $\mathcal{G}$ - and $\mathcal{V}$-optimal, we construct the profile sets in such a way that the information matrix (8) is diagonal with elements that are as large as possible. The approach was motivated by Goos (2006b) who presented conditions for designing optimal two-level main-effect split-plot experiments. To find the conjoint design $\mathbf{X}$ corresponding to a diagonal information matrix $\mathbf{X}^{\prime} \mathbf{V}^{-1} \mathbf{X}$ with the largest possible diagonal elements, both matrices $\mathbf{A}$ and $\mathbf{B}$ in (9) should be diagonal. The diagonal elements of $\mathbf{A}$ should be as large as possible, whereas those of $\mathbf{B}$ should be as small as possible.

In our construction method we present in Section $5, \mathbf{X}$ will turn out to be an orthogonal array such as a full $2^{k}$ or fractional $2^{k-p}$ factorial design or a Plackett-Burman design. As a result, $\mathbf{A}=n \mathbf{I}_{k+1}$. Ideally, the profiles in the orthogonal design $\mathbf{X}$ are arranged in sets so that $\mathbf{B}$ is a zero matrix. However, because we are concerned with experiments with large numbers of attributes where some levels are held constant to simplify the respondents' task, this is impossible. Also, the first element of $\mathbf{B}$ corresponds to the intercept and equals $b m^{2}$ for every imaginable design. How best to assign these constant attribute levels is discussed in the next section.

\section{Large numbers of attributes}

In order not to overload the respondents with a heavy rating task, we hold one or more attributes at a fixed level in each of the profile sets. In other words, we introduce perfect level overlap for one or more attributes in each profile set. These attributes are the constant attributes and may differ from set to set. We denote the number of constant 
attributes by $k_{c}$. The remaining $k_{v}=k-k_{c}$ attributes, the levels of which may vary, are the non-constant attributes. The concepts of constant and non-constant attributes are illustrated with the conjoint design in Table 1. This design consists of $b=12$ sets with $m=2$ profiles each. It has $k=6$ attributes, two of which are constant so that $k_{c}=2$ and $k_{v}=4$. The levels of the constant attributes are highlighted in grey. As can be seen, the constant and non-constant attributes may differ between the profile sets.

Table 1: Optimal variance-balanced conjoint design with $b=12$ sets of $m=2$ profiles, $k_{c}=2$ constant attributes and $k_{v}=4$ non-constant attributes.

\begin{tabular}{|c|cccccc|}
\hline & \multicolumn{7}{|c|}{ Attributes } \\
Set & 1 & 2 & 3 & 4 & 5 & 6 \\
\hline 1 & -1 & -1 & -1 & -1 & -1 & -1 \\
1 & -1 & +1 & +1 & -1 & +1 & +1 \\
\hline 2 & -1 & -1 & -1 & +1 & +1 & +1 \\
2 & -1 & +1 & +1 & +1 & -1 & -1 \\
\hline 3 & +1 & -1 & +1 & -1 & -1 & +1 \\
3 & +1 & +1 & -1 & -1 & +1 & -1 \\
\hline 4 & +1 & -1 & +1 & -1 & +1 & -1 \\
4 & +1 & +1 & -1 & +1 & -1 & +1 \\
\hline 5 & -1 & -1 & -1 & -1 & -1 & +1 \\
5 & +1 & -1 & +1 & +1 & -1 & -1 \\
\hline 6 & -1 & -1 & -1 & +1 & +1 & -1 \\
6 & +1 & -1 & +1 & -1 & +1 & +1 \\
\hline 7 & -1 & +1 & +1 & -1 & -1 & -1 \\
7 & +1 & +1 & -1 & +1 & -1 & +1 \\
\hline 8 & -1 & +1 & +1 & +1 & +1 & +1 \\
8 & +1 & +1 & -1 & -1 & +1 & -1 \\
\hline 9 & -1 & -1 & -1 & -1 & -1 & -1 \\
9 & +1 & +1 & -1 & +1 & +1 & -1 \\
\hline 10 & -1 & -1 & -1 & +1 & +1 & +1 \\
10 & +1 & +1 & -1 & -1 & -1 & +1 \\
\hline 11 & -1 & +1 & +1 & -1 & +1 & -1 \\
11 & +1 & -1 & +1 & +1 & -1 & -1 \\
\hline 12 & -1 & +1 & +1 & +1 & -1 & +1 \\
12 & +1 & -1 & +1 & -1 & +1 & +1 \\
\hline
\end{tabular}

Keeping the levels of one or more attributes fixed in the profile sets of a conjoint design reduces the amount of information that can be collected from the experiment. Also Street et al. (2001) observed that imposing perfect level overlap in two-level main-effect paired comparison designs leads to information losses with respect to the part-worths of the constant attributes in each of the profile sets. Without perfect level overlap constraints, most information is obtained when the levels of each attribute are maximum balanced, 
meaning that they occur with equal frequency in each profile set. Note that this is the opposite of perfect level overlap.

If no constant attributes were involved, maximum level balance would imply that $\mathbf{X}_{i}^{\prime} \mathbf{1}_{m}=\left[m \mathbf{0}_{1 \times k}\right]^{\prime}, i=1, \ldots, b$, so that all elements of the matrix $\mathbf{B}$ in (9) are zero, except for the single element corresponding to the intercept in the upper left entry. This would maximize the information in the experiment. When $k_{c}$ constant attributes are imposed on the design, $\mathbf{X}_{i}^{\prime} \mathbf{1}_{m}=\left[m \mathbf{s}_{i}^{\prime}\right]^{\prime}, i=1, \ldots, b$, where $\mathbf{s}_{i}$ is a $k \times 1$ vector containing at least $k_{c}$ nonzero elements. As a result of that, $\mathbf{B}$ has additional nonzero elements that cause the loss of information. To minimize the number of nonzero elements in $\mathbf{B}$, the profiles in $\mathbf{X}$ forming an orthogonal array should be grouped such that maximum level balance is still preserved for the non-constant attributes in each profile set. In that case, $\mathbf{s}_{i}$ has exactly $k_{c}$ nonzero elements. In the conjoint design of Table 1 , the profiles represent a Plackett-Burman design and their grouping is characterized by maximum level balance for the non-constant attributes.

It is possible that the optimal designs do not spread the information losses from perfect level overlap evenly over all part-worths. Stated differently, the diagonal elements for the part-worths of the matrix $\mathbf{B}$ in (9), and thus of the information matrix (8), may not be the same. Still, we assume that all part-worths are equally important so that we look for optimal designs that yield the same amount of information on each part-worth. Each attribute should therefore be constant in an equal number of profile sets. Optimal designs that meet this requirement are called variance-balanced. The conjoint design of Table 1 is optimal and variance-balanced. As can be seen, each attribute acts as a constant attribute in four profile sets.

The fact that each attribute in the conjoint design of Table 1 is constant in an even number of profile sets is not surprising. This is generally the case for optimal variancebalanced conjoint designs. As we choose $\mathbf{X}$ to be an orthogonal array and arrange the profiles so that the levels of each non-constant attribute sum to zero in each profile set, the levels of each constant attribute necessarily sum to zero over the profile sets. This implies an even number of profile sets for each constant attribute to offset a -1 for a constant attribute in a profile set by $\mathrm{a}+1$ for the same constant attribute in another profile set.

Now, with this framework in mind, we describe our strategy to set up optimal twolevel variance-balanced conjoint designs in which a number of attributes are constant in each profile set. Compared with optimal designs without constant attributes, these designs give up some statistical efficiency to keep the rating tasks doable. Also, to show manageable profile sets, we disregard designs with more than $k_{c}=10$ constant attributes or more than $k_{v}=4$ non-constant attributes. To develop the optimal variance-balanced conjoint designs, we need to determine 
1. the constant attributes in each profile set,

2. the levels of these constant attributes and

3. the levels of the non-constant attributes.

In the next section, we discuss our design construction approach taking into account this sequence of steps.

\section{Design construction approach}

In our design construction approach, we distinguish between $k_{c}=1$ and $k_{c}>1$ constant attributes. For both cases, we run through the three steps as listed above. We explain our strategy by some example designs that appear in Appendix A. Optimal variance-balanced conjoint designs for $k_{c}=1$ and $k_{c}>1$ appear in Appendix A.1 and Appendix A.2, respectively.

\subsection{Optimal variance-balanced conjoint designs for $k_{c}=1$}

The optimal variance-balanced designs with $k_{c}=1$ constant attribute in Appendix A.1 are the smallest ones that can be created for $k_{v}=2,3$ or 4 non-constant attributes and $m=2,4$ or 8 profiles per set. To construct these designs, we cover the three steps as follows.

\subsubsection{Steps 1 \& 2: Determining the constant attributes and their levels}

When $k_{c}=1$, determining the constant attributes and their levels is fairly straightforward. To allow for variance balance, each attribute should be constant in an equal number of profile sets. Also, each attribute should be constant in an even number of profile sets to have as many -1 's as +1 's for their levels. In the optimal variance-balanced conjoint designs of Tables A.1, A.2, A.4, A.5, A.7 and A.8 each attribute is constant in two profile sets and in the designs of Tables A.3 and A.6 each attribute is constant in four profile sets.

\subsubsection{Step 3: Determining the levels of the non-constant attributes}

To determine the levels of the non-constant attributes, we draw on orthogonal subdesigns. For $k_{v}=2,3$ or 4 non-constant attributes and $m=2,4$ or 8 profiles per set, we constructed the smallest possible orthogonal subdesigns with an even number of profile sets. As such, we can match these subdesigns with the constant attributes to produce the conjoint designs of Appendix A.1. We illustrate the use of orthogonal subdesigns for each case. 
The optimal variance-balanced conjoint designs in Tables A.1 and A.2 have $k_{v}=2$ non-constant attributes and profile sets of sizes $m=2$ and $m=4$, respectively. The design in Table A.1 is built on the orthogonal subdesign in Table 2a and the design in Table A.2 is built on the orthogonal subdesign in Table $2 \mathrm{~b}$. The subdesign in Table $2 \mathrm{a}$ consists of two profile sets of size $m=2$ and the subdesign in Table $2 \mathrm{~b}$ consists of two profile sets of size $m=4$. The former subdesign and each of the profile sets in the latter subdesign represent the full $2^{2}$ factorial design.

Table 2: Orthogonal subdesigns with the levels of $k_{v}=2$ non-constant attributes. The subdesigns consist of two profile sets each.

\begin{tabular}{|c|cc|}
\multicolumn{3}{c}{ a) } \\
\hline & NC & attr \\
Set & 1 & 2 \\
\hline 1 & -1 & -1 \\
1 & +1 & +1 \\
\hline 2 & -1 & +1 \\
2 & +1 & -1 \\
\hline
\end{tabular}

\begin{tabular}{|c|c|c|}
\hline \multicolumn{3}{|c|}{ b) $m=4$} \\
\hline & $\mathrm{NC}$ & attr \\
\hline Set & 1 & 2 \\
\hline 1 & -1 & -1 \\
\hline 1 & +1 & +1 \\
\hline 1 & -1 & +1 \\
\hline 1 & +1 & -1 \\
\hline 2 & -1 & -1 \\
\hline 2 & +1 & +1 \\
\hline 2 & -1 & +1 \\
\hline 2 & +1 & -1 \\
\hline
\end{tabular}

The subdesigns in Tables $2 \mathrm{a}$ and $2 \mathrm{~b}$ are incorporated thrice in the conjoint designs of Tables A.1 and A.2, respectively. Since this is the required minimum to have each of the $k=3$ attributes act as a constant attribute, the conjoint designs are the smallest ones that can be produced. They both involve six respondents, but can be replicated to set up larger conjoint designs in which the number of respondents is a multiple of six. The resulting designs are still optimal and variance-balanced. Note that we have not shown an optimal variance-balanced conjoint design with $k_{v}=2$ non-constant attributes and sets of $m=8$ profiles. This is because the profile sets in such design would contain only duplicated profiles which makes no sense in a conjoint study.

The optimal variance-balanced conjoint designs with $k_{c}=1$ constant attribute in Tables A.3, A.4 and A.5 have $k_{v}=3$ non-constant attributes and profile sets of sizes $m=2,4$ and 8 , respectively. The orthogonal subdesigns needed for their construction appear in Tables 3a, 3b and 3c. The subdesign in Table 3a embraces four profile sets of size $m=2$ and the subdesigns in Tables $3 \mathrm{~b}$ and $3 \mathrm{c}$ embrace two profile sets of sizes $m=4$ and $m=8$. The full $2^{3}$ factorial design was exploited to build the subdesigns. It is included once in the subdesigns of Tables $3 \mathrm{a}$ and $3 \mathrm{~b}$ and twice in the subdesign of Table 3c.

The subdesigns are incorporated four times in the conjoint designs of Tables A.3, A.4 and A.5 to allow each of the $k=4$ attributes to act as a constant attribute. As such, the conjoint designs are the smallest possible ones. For the design in Table A.3 sixteen 
Table 3: Orthogonal subdesigns with the levels of $k_{v}=3$ non-constant attributes. Subdesign a) consists of four profile sets, while subdesigns b) and c) consist of two profile sets.

\begin{tabular}{|c|ccc|}
\multicolumn{3}{c}{ a) $m=2$} \\
\hline & \multicolumn{3}{|c|}{ NC attr } \\
Set & 1 & 2 & 3 \\
\hline 1 & -1 & -1 & -1 \\
1 & +1 & +1 & +1 \\
\hline 2 & -1 & -1 & +1 \\
2 & +1 & +1 & -1 \\
\hline 3 & -1 & +1 & -1 \\
3 & +1 & -1 & +1 \\
\hline 4 & -1 & +1 & +1 \\
4 & +1 & -1 & -1 \\
\hline
\end{tabular}

\begin{tabular}{|c|ccc|}
\multicolumn{1}{c}{ b) $m=4$} \\
\hline & \multicolumn{3}{|c}{ NC attr } \\
Set & 1 & 2 & 3 \\
\hline 1 & -1 & -1 & -1 \\
1 & +1 & +1 & +1 \\
1 & -1 & -1 & +1 \\
1 & +1 & +1 & -1 \\
\hline 2 & -1 & +1 & -1 \\
2 & +1 & -1 & +1 \\
2 & -1 & +1 & +1 \\
2 & +1 & -1 & -1 \\
\hline
\end{tabular}

\begin{tabular}{|c|ccc|}
\multicolumn{3}{c}{ c) $m=8$} \\
\hline & \multicolumn{3}{|c}{ NC attr } \\
Set & 1 & 2 & 3 \\
\hline 1 & -1 & -1 & -1 \\
1 & +1 & +1 & +1 \\
1 & -1 & -1 & +1 \\
1 & +1 & +1 & -1 \\
1 & -1 & +1 & -1 \\
1 & +1 & -1 & +1 \\
1 & -1 & +1 & +1 \\
1 & +1 & -1 & -1 \\
\hline 2 & -1 & -1 & -1 \\
2 & +1 & +1 & +1 \\
2 & -1 & -1 & +1 \\
2 & +1 & +1 & -1 \\
2 & -1 & +1 & -1 \\
2 & +1 & -1 & +1 \\
2 & -1 & +1 & +1 \\
2 & +1 & -1 & -1 \\
\hline
\end{tabular}

respondents are needed and for the designs in Tables A.4 and A.5 eight respondents are needed. To build larger optimal variance-balanced conjoint designs with $k_{c}=1$ constant attribute and $k_{v}=3$ non-constant attributes, replications of the design tables should be made.

The last optimal variance-balanced conjoint designs with $k_{c}=1$ constant attribute are the ones in Tables A.6, A.7 and A.8 containing $k_{v}=4$ non-constant attributes and profile sets of sizes $m=2,4$ and 8 , respectively. The designs are constructed using the orthogonal subdesigns listed in Tables 4a, 4b and 4c. Table 4a shows two possible subdesigns of four profile sets of size $m=2$ and Table $4 \mathrm{~b}$ shows two possible subdesigns of two profile sets of size $m=4$. The subdesign in Table $4 \mathrm{c}$ has two profile sets of size $m=8$. The combinations of the two possible subdesigns in Tables 4a and $4 \mathrm{~b}$ and the subdesign in Table $4 \mathrm{c}$ represent the full $2^{4}$ factorial design. The subdesigns in Tables $4 \mathrm{a}$ and $4 \mathrm{~b}$ each constitute an orthogonal fraction of this design.

We produced the conjoint design in Table A.6 by implementing the two possible subdesigns in Table 4a one after the other. In the same way, we constructed the conjoint design in Table A.7 from the two subdesigns in Table 4b. Other replication structures of the subdesigns are also possible since the optimality of the designs is not affected by the choice of subdesigns used. The three conjoint designs are the smallest possible ones 
Table 4: Orthogonal subdesigns with the levels of $k_{v}=4$ non-constant attributes. Subdesigns i. and ii. of a) consist of four profile sets, while subdesigns i. and ii. of b) and subdesign c) consist of two profile sets.

a) $m=2$

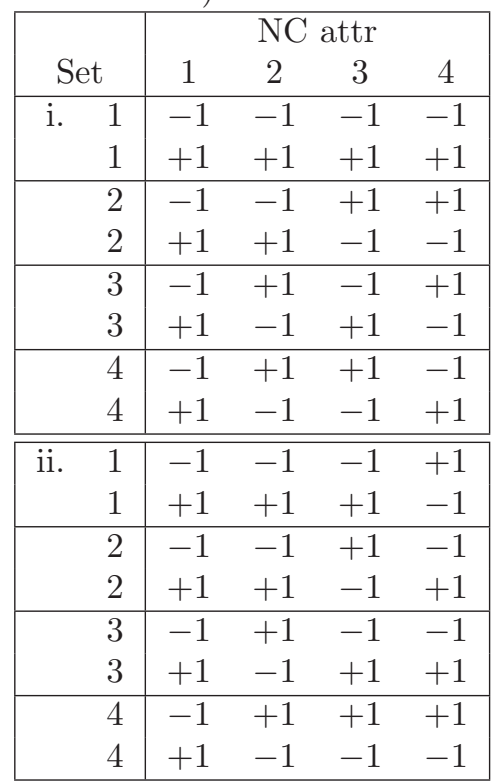

b) $m=4$

\begin{tabular}{|cc|cccc|}
\hline \multicolumn{2}{|c|}{ Set } & 1 & 2 & 3 & 4 \\
\hline i. & 1 & -1 & -1 & -1 & -1 \\
& 1 & +1 & +1 & +1 & +1 \\
& 1 & -1 & -1 & +1 & +1 \\
& 1 & +1 & +1 & -1 & -1 \\
\hline & 2 & -1 & +1 & -1 & +1 \\
& 2 & +1 & -1 & +1 & -1 \\
& 2 & -1 & +1 & +1 & -1 \\
& 2 & +1 & -1 & -1 & +1 \\
\hline \hline ii. & 1 & -1 & -1 & -1 & +1 \\
& 1 & +1 & +1 & +1 & -1 \\
& 1 & -1 & -1 & +1 & -1 \\
& 1 & +1 & +1 & -1 & +1 \\
\hline & 2 & -1 & +1 & -1 & -1 \\
2 & +1 & -1 & +1 & +1 \\
2 & -1 & +1 & +1 & +1 \\
2 & +1 & -1 & -1 & -1 \\
\hline
\end{tabular}

c) $m=8$

\begin{tabular}{|c|c|c|c|c|}
\hline \multirow[b]{2}{*}{ Set } & \multicolumn{4}{|c|}{$\mathrm{NC}$ attr } \\
\hline & 1 & 2 & 3 & 4 \\
\hline 1 & -1 & -1 & -1 & -1 \\
\hline 1 & +1 & +1 & +1 & +1 \\
\hline 1 & -1 & -1 & +1 & +1 \\
\hline 1 & +1 & +1 & -1 & -1 \\
\hline 1 & -1 & +1 & -1 & +1 \\
\hline 1 & +1 & -1 & +1 & -1 \\
\hline 1 & -1 & +1 & +1 & -1 \\
\hline 1 & +1 & -1 & -1 & +1 \\
\hline 2 & -1 & -1 & -1 & +1 \\
\hline 2 & +1 & +1 & +1 & -1 \\
\hline 2 & -1 & -1 & +1 & -1 \\
\hline 2 & +1 & +1 & -1 & +1 \\
\hline 2 & -1 & +1 & -1 & -1 \\
\hline 2 & +1 & -1 & +1 & +1 \\
\hline 2 & -1 & +1 & +1 & +1 \\
\hline 2 & +1 & -1 & -1 & -1 \\
\hline
\end{tabular}

since five subdesigns are needed to allow each of the $k=5$ attributes to act as a constant attribute. The design in Table A.6 requires twenty respondents and the designs in Tables A.7 and A.8 require ten respondents. The designs can be replicated when larger conjoint experiments with $k_{c}=1$ constant attribute and $k_{v}=4$ non-constant attributes are desirable.

\subsection{Optimal variance-balanced conjoint designs for $k_{c}>1$}

The optimal variance-balanced conjoint designs with $k_{c}>1$ constant attributes in Appendix A.2 appear in the right panels of the tables. The other panels are meant to clarify our design construction approach. The conjoint design in the right panel of Table A.9 is the same design as in Table 1 for $k_{c}=2$ constant attributes, $k_{v}=4$ non-constant attributes and $m=2$ profiles per set. The next two designs in Tables A.10 and A.11 extend the starting example to $m=4$ and $m=8$ profiles per set, respectively. We first explain the main idea of our strategy using these three conjoint designs and then discuss the other conjoint designs of Appendix A.2.

\subsubsection{Step 1: Determining the constant attributes}

If $k_{c}>1$, it may be quite cumbersome to look for combinations of constant attributes that lead to perfect level overlap of each attribute in an equal number of profile sets. 
This is particularly true when $k_{c}$ is large and not a divisor of $k$. Therefore, we advocate the use of balanced or partially balanced incomplete block designs (BIBDs or PBIBDs) to determine patterns of constant attributes that result in variance-balanced conjoint designs. BIBDs and PBIBDs describe how to arrange the levels of a single qualitative factor, called treatments, in groups or blocks of a certain size. Each treatment thereby occurs an equal number of times in the entire design. In BIBDs, the number of times two different treatments occur together in a block is the same for all pairs of treatments. This is not true for PBIBDs which makes there are more and smaller PBIBDs than BIBDs for a given number of treatments and block size.

We refer to the work of Cochran and Cox (1957) and Cox (1958) for a general account of BIBDs and PBIBDs. The former authors depict some tables of BIBDs whereas the latter author describes a simple method to set up PBIBDs. More discussions and tables of BIBDs are provided by Abel and Greig (1996) and Mathon and Rosa (1996). PBIBDs are fully elaborated in the work of Shah and Sinha (1989) and Street and Street (1996), and catalogs of these designs can be found in the work of Clatworthy (1973) and on a website by Sinha (see the References).

In Tables 5a, 5b, 5c and 5d, three PBIBDs and one BIBD are listed for six treatments and block sizes of two. Each of these designs can be exploited to construct optimal variance-balanced conjoint designs with $k_{c}=2$ constant attributes and $k_{v}=4$ nonconstant attributes. Each block in the PBIBDs and the BIBD determines which of the six attributes should be constant in a prespecified number of profile sets. So each block defines $k_{c}=2$ constant attributes for a certain number of profile sets. Because each of the six treatments appears the same number of times in the PBIBDs and the BIBD, each of the $k=6$ attributes is constant in an equal number of profile sets.

The conjoint designs in Tables A.9, A.10 and A.11 are built on the PBIBD of Table 5a. This is illustrated in the left panels of the tables. The PBIBD has three blocks of size two indicating three combinations of two constant attributes. The first block of the PBIBD tells us that Attributes 1 and 4 should be constant in some of the profile sets. This is done in Profile sets 1 to 4 for each of the conjoint designs. The second block determines Attributes 2 and 5 to be constant. These constant attributes are exploited in Profile sets 5 to 8 . According to the last block, Attributes 3 and 6 should be constant. This is accomplished in Profile sets 9 to 12. So each block of constant attributes is embedded in

four profile sets in each of the conjoint designs. How to decide on the number of profile sets in which the same attributes are constant is laid out in the next section.

\subsubsection{Steps $2 \&$ 3: Determining the levels of the constant and non-constant attributes}

The optimal variance-balanced conjoint designs in Appendix A.2 are all developed from PBIBDs and are the smallest ones that can be produced. For the conjoint designs in Tables A.9, A.10 and A.11, we showed that the constant attributes are dictated by the three blocks in the PBIBD of Table 5a. The question now is at what levels these attributes 
Table 5: a) PBIBD with 3 blocks, b) PBIBD with 9 blocks, c) PBIBD with 12 blocks and d) BIBD with 15 blocks. Each design has 6 treatments and block sizes of 2 .



have to be set and how often they have to be held fixed. To answer this question, we use intermediate designs, so-called constant attribute designs that are orthogonal for the constant attributes. These constant attribute designs appear in the middle panel of each design table.

To construct the constant attribute designs, we exploit the full $2^{k_{c}}$ factorial design or an orthogonal fraction of the full $2^{k_{c}}$ factorial design. The sample size of the full $2^{k_{c}}$ factorial design or the orthogonal fraction then determines the number of profile sets for each combination of constant attributes. The constant attribute designs for the conjoint designs in Tables A.9, A.10 and A.11 utilize the full $2^{2}$ factorial design for each combination of constant attributes. This means that $2^{2}=4$ profile sets have the same attributes constant with levels that fit the full $2^{2}$ factorial design.

The last problem to solve before the design construction is complete is the determination of the levels of the non-constant attributes. Therefore, we call on the orthogonal subdesigns for the $k_{v}$ non-constant attributes given by the tables in Section 5.1.2. In the conjoint design of Table A.9, the two possible subdesigns of Table 4a are implemented. Since three subdesigns can be accommodated, one subdesign is implemented once and the other twice. They nicely fit in the four profile sets from the full $2^{2}$ factorial design corresponding to a combination of constant attributes. Similarly, in the conjoint design of Table A.10 each of the subdesigns in Table $4 \mathrm{~b}$ is implemented thrice and in the conjoint design of Table A.11 the subdesign in Table 4c is implemented six times. 
In some cases if $k_{c}=2$, the two non-orthogonal fractions of the full $2^{2}$ factorial design in Table 6 provide another way to determine the levels of the constant attributes. These fractions require only two profile sets for each combination of constant attributes and thus allow for smaller conjoint designs when $k_{c}=2$. They both have one attribute at a fixed level so that they need to be jointly implemented to offset the fixed level of -1 by the fixed level of +1 . As a result, the fractions can only be employed if the number of blocks in the $\mathrm{BIBD}$ or PBIBD is even and the orthogonal subdesigns for the $k_{v}$ non-constant attributes enclose two profile sets. The use of the fractions is illustrated in the conjoint designs of Tables A.12 and A.13 for $k_{c}=2$ constant attributes, $k_{v}=2$ non-constant attributes and $m=2$ and $m=4$ profiles per set, respectively. The PBIBD specifies four combinations of constant attributes, the levels of which are determined by the non-orthogonal fractions. The subdesigns in Tables $2 \mathrm{a}$ and $2 \mathrm{~b}$ fill out the levels of the non-constant attributes.

Table 6: Non-orthogonal fractions of the full $2^{2}$ factorial design for constructing constant attribute designs for $k_{c}=2$ constant attributes.

ii \begin{tabular}{c|c|cc|}
\hline & \multicolumn{2}{|c|}{ Cst } & attr \\
ii & Set & 1 & 2 \\
\cline { 2 - 4 } & 1 & -1 & -1 \\
2 & -1 & +1 \\
\hline 1 & +1 & -1 \\
2 & +1 & +1 \\
\hline
\end{tabular}

To further illustrate our design construction approach, we discuss the optimal variancebalanced conjoint designs in Tables A.14 and A.15. They both have $m=2$ profiles per set. The design in Table A.14 has $k_{c}=3$ constant attributes and $k_{v}=3$ non-constant attributes and the design in Table A.15 has $k_{c}=4$ constant attributes and $k_{v}=4$ nonconstant attributes. To fix the levels of the constant attributes, minimum-size orthogonal fractions of the full $2^{k_{c}}$ factorial design are incorporated. These fractions are the smallest possible ones that allow the estimation of all $k_{c}$ main effects. If $3 \leq k_{c} \leq 10$, minimum-size orthogonal fractions for the $k_{c}$ constant attributes can be constructed using the generators in Table 7. We selected these generators from Appendix 4A on pages 193-194 of Wu and Hamada (2000). Other generators that yield larger orthogonal fractions can also be retrieved in this appendix.

The orthogonal fraction has a size of $2^{k_{c}-p}$, where $2^{-p}$ refers to the fraction of the full $2^{k_{c}}$ factorial design. The full $2^{k_{c}-p}$ factorial design is used to determine the levels of the first $k_{c}-p$ constant attributes. The levels of the remaining $p$ constant attributes are specified by the generators. For example, in case $k_{c}=3$, the generator is $3=12$ meaning that the level of the third constant attribute is obtained by multiplying the levels of the first and second constant attribute. In this way, one orthogonal fraction is constructed. Other orthogonal fractions of the full $2^{k_{c}}$ factorial design can be produced by using one or more generators with a minus sign. For $k_{c}=3,3=-12$ is the generator of the second 
Table 7: Generators for constructing minimum-size orthogonal fractions for $k_{c}$ attributes.

\begin{tabular}{|c|c|l|}
\hline$k_{c}$ & Size & \multicolumn{1}{|c|}{ Generators } \\
\hline 3 & $2^{3-1}=4$ & $3=12$ \\
4 & $2^{4-1}=8$ & $4=123$ \\
5 & $2^{5-2}=8$ & $4=12,5=13$ \\
6 & $2^{6-3}=8$ & $4=12,5=13,6=23$ \\
7 & $2^{7-4}=8$ & $4=12,5=13,6=23,7=123$ \\
8 & $2^{8-4}=16$ & $5=123,6=124,7=134,8=234$ \\
9 & $2^{9-5}=16$ & $5=123,6=124,7=134,8=234,9=1234$ \\
10 & $2^{10-6}=16$ & $5=123,6=124,7=134,8=234,9=1234,10=34$ \\
\hline
\end{tabular}

and only remaining orthogonal fraction.

We derive from Table 7 that there are also two minimum-size orthogonal fractions for $k_{c}=4$ constant attributes. In the constant attribute design of Table A.14 each of two minimum-size orthogonal fractions for $k_{c}=3$ constant attributes is used twice and in the constant attribute design of Table A.15 each of two minimum-size orthogonal fractions for $k_{c}=4$ constant attributes is used thrice. The orthogonal fractions for $k_{c}=3$ involve $2^{3-1}=4$ profile sets for each combination of constant attributes and the orthogonal fractions for $k_{c}=4$ involve $2^{4-1}=8$ profile sets. The levels of the non-constant attributes in the conjoint designs are set by means of the subdesign in Table $3 \mathrm{a}$ for $k_{v}=3$ and the two possible subdesigns in Table $4 \mathrm{a}$ for $k_{v}=4$.

\section{Information content of the optimal variance-bal- anced conjoint designs}

For our study, we examine the designs in Appendix A that have $k=4$ attributes. These are the designs in Tables A.3, A.4 and A.5 of Appendix A.1 for $k_{c}=1$ constant attribute and $k_{v}=3$ non-constant attributes and the designs in Tables A.12 and A.13 of Appendix A.2 for $k_{c}=2$ constant attributes and $k_{v}=2$ non-constant attributes. The designs with $k_{c}=1$ constant attribute have profile sets of sizes $m=2,4$ and 8 , respectively, and the designs with $k_{c}=2$ constant attributes have profile sets of sizes $m=2$ and $m=4$. We compare the information content of these designs with that of the optimal conjoint designs in which no constant attributes are used. These latter conjoint designs are nothing but orthogonally blocked two-level designs. They can be constructed using the generators in Appendix 3A on pages 150-151 and Appendix 4B on pages 199-203 of Wu and Hamada (2000).

In general, the information matrix (8) for optimal variance-balanced conjoint designs is diagonal with maximal diagonal elements that are equal for the part-worths. In Ap- 
pendix B, we computed the information matrix for the design in Table A.12. Based on these computations, we derived the amount of information on the intercept and partworths for the other conjoint designs. The results for all five conjoint designs appear in Table 8.

For a given design setting, the optimal conjoint design without constant attributes leads to the same amount of information on the intercept as provided by the optimal conjoint design with constant attributes. However, as we mentioned in Sections 3 and 4, the amount of information on each part-worth of the optimal designs without constant attributes is larger. Because the levels of each attribute are maximum balanced in each of the profile sets, the part-worth elements of the matrix $\mathbf{B}$ in (9) are zero. As a result, the amount of information on each part-worth of the optimal designs without constant attributes is equal to $n$, the number of design profiles. For each of the five design cases in Table 8, we compared the value of $n$ with the values for the part-worths in the table for 10 degrees of correlation $\rho \in\{0 ; 0.1 ; \ldots ; 0.9\}$. As such, we computed the percentage information losses for each part-worth from imposing constant attributes. We plotted them in Figure 1.

Table 8: Amount of information on the intercept and part-worths of the optimal variancebalanced conjoint designs for a) $k_{c}=1$ and $k_{v}=3$ contained in Tables A.3, A.4 and A.5 and for b) $k_{c}=k_{v}=2$ contained in Tables A.12 and A.13.

\begin{tabular}{r|cccc|cc|}
\cline { 2 - 7 } & \multicolumn{3}{c|}{ Conjoint design } & \multicolumn{2}{c|}{ Information on } \\
a) $k_{c}=1$ & Table & $m$ & $b$ & $n$ & the intercept & each part-worth \\
\cline { 2 - 7 }$k_{v}=3$ & A.3 & 2 & 16 & 32 & $32(1-\rho) /(1+\rho)$ & $8(1-\rho) /(1+\rho)+24$ \\
b) $k_{c}=2$ & A.4 & 4 & 8 & 32 & $32(1-\rho) /(1+3 \rho)$ & $8(1-\rho) /(1+3 \rho)+24$ \\
$k_{v}=2$ & A. 12 & 8 & 8 & 64 & $64(1-\rho) /(1+7 \rho)$ & $16(1-\rho) /(1+7 \rho)+48$ \\
\cline { 2 - 7 } & A.13 & 4 & 8 & 16 & $16(1-\rho) /(1+\rho)$ & $8(1-\rho) /(1+\rho)+8$ \\
\cline { 2 - 6 } & & & & $32(1-\rho) /(1+3 \rho)$ & $16(1-\rho) /(1+3 \rho)+16$ \\
\hline
\end{tabular}

A close look at Figure 1 reveals that, given $m=2$ or $m=4$ and a value of $\rho$, the information losses for each part-worth of the conjoint designs with $k_{c}=2$ constant attributes are twice the information losses of the conjoint designs with $k_{c}=1$ constant attribute. We verified with some additional computations that in general, if the number of attributes, $k$, is fixed in a series of optimal two-level variance-balanced conjoint designs, the information losses are proportional to the number of constant attributes, $k_{c}$, used.

Figure 1 also shows that, given $k_{c}$ and $\rho$, the information losses for each part-worth increase with $m$. As the profile sets of the conjoint designs get larger or the number of respondents drops, the information losses from constant attributes increase. This can also be concluded from $c_{8}<c_{4}<c_{2}<1$, where $c_{m}=(1-\rho) /(1+(m-1) \rho)$ (see Appendix B). It implies that for a given number of design profiles, $n$, sets with $m=2$ profiles are more efficient than sets with $m=4$ profiles, which in their turn, are more efficient than sets 


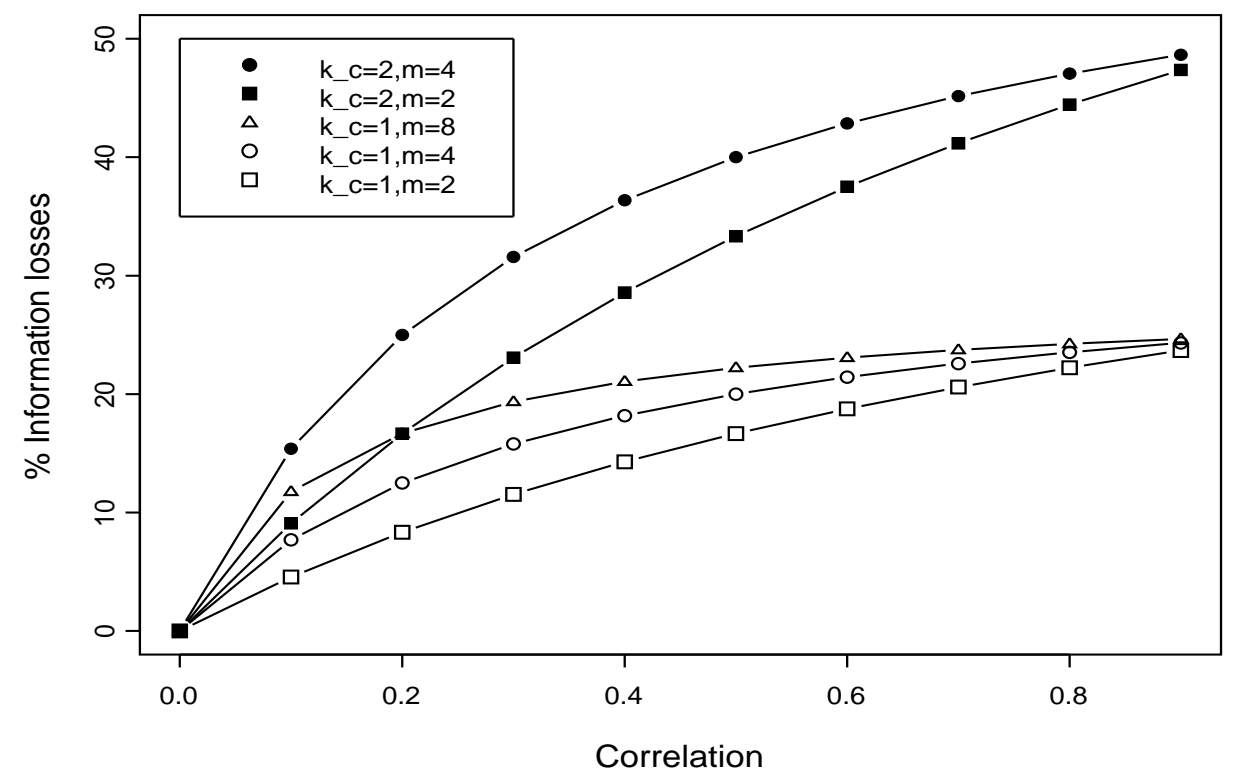

Figure 1: \% Information losses for each part-worth from using $k_{c}=1$ and $k_{c}=2$ constant attributes for degrees of correlation ranging from 0 to 0.9. The five designs of Table 8 are considered.

with $m=8$ profiles. For example, the designs in Tables A.3 and A.4 have equal sample sizes and equal numbers of profiles in which each attribute is constant. However, because the former design is constructed for $m=2$ and the latter for $m=4$, the information losses of the latter design are larger.

A last observation from Figure 1 is that, given $m$ and $k_{c}$, the information losses for each part-worth increase with $\rho$. The more heterogeneous respondents are, the more information one loses by keeping the levels of one or more attributes constant. The increase of the losses with $\rho$ is fairly linear if $m=2$, but becomes quadratic for larger values of $m$. Note that if $\rho=0$, respondents are assumed to be homogeneous so that the grouping of the profiles in sets, and thus also the matrix $\mathbf{B}$ in (9), does not matter anymore.

\section{Conclusion}

In this chapter, we presented a simple approach to construct optimal two-level conjoint designs that involve a large number of attributes. To reduce the cognitive burden on the respondents, the designs hold the levels of one or more attributes constant in each of the profile sets. The designs have sets of 2,4 or 8 profiles and are optimal with respect to the $\mathcal{D}-, \mathcal{A}-, \mathcal{G}$ - and $\mathcal{V}$-optimality criteria for estimating main-effects models. 
The optimal conjoint designs have a diagonal information matrix collecting maximum information. In addition, the conjoint designs are variance-balanced meaning that they yield an equal amount of information on each of the part-worths. Also, the conjoint designs do not depend on the extent to which respondents are heterogeneous, as expressed by the degree of correlation. This makes them very practical to use. Prior to constructing the conjoint designs, we advise practitioners to think carefully about the number of constant attributes they want. Compared with optimal conjoint designs without constant attributes, optimal conjoint designs with constant attributes lead to information losses that are proportional to the number of constant attributes.

If more than one constant attribute is desirable, our design construction method draws on BIBDs and PBIBDs to provide patterns of constant attributes that allow for variance balance. To ensure optimality, we choose the levels of the $k_{c}$ constant attributes and the levels of the $k_{v}$ non-constant attributes such that they are orthogonal for each combination of constant attributes. We first determine the levels of the $k_{c}$ constant attributes by incorporating the full $2^{k_{c}}$ factorial design or an orthogonal fraction of it. We then set the levels of the $k_{v}$ non-constant attributes by using a prespecified orthogonal subdesign for the $k_{v}$ non-constant attributes.

The design examples provided in Appendix A are the smallest ones supported by our approach. There are generally three ways to obtain larger conjoint designs. A first option is to choose a larger BIBD or PBIBD that defines more combinations of constant attributes. A second possibility is to employ larger orthogonal designs to fix the levels of the $k_{c}$ constant attributes. Lastly, a small conjoint design may be replicated.

As an additional justification of our method, it is interesting to note that the optimal variance-balanced conjoint designs with sets of two profiles are similar to the variance-balanced constant difference pairs constructed by Severin (2000). For example, the optimal paired comparison design with four attribute level differences in the work of Severin (2000, page 142) is equivalent to the duplicated conjoint design of our starting example in Table 1. In other words, Severin's (2000, page 142) design has the same structure as the conjoint design in Table 1 but contains twice as many profile sets.

Finally, more work is needed to extend our method for constructing optimal two-level variance-balanced conjoint designs with sets of 3,5,6 or 7 profiles. Also the production of conjoint designs using models with main effects plus interactions might be considered. 


\section{Appendix A. Optimal variance-balanced conjoint de- signs}

\section{A.1. Designs with one constant attribute: $k_{c}=1$}

Table A.1: Smallest optimal variance-balanced conjoint design with sets of $m=2$ profiles, $k_{c}=1$ constant attribute and $k_{v}=2$ non-constant attributes.

\begin{tabular}{|c|ccc||c|ccc||cc|cc|}
\hline & \multicolumn{4}{|c|}{ Attributes } & \multicolumn{4}{c|}{ Attributes } & \multicolumn{3}{c|}{ Attributes } \\
Set & 1 & 2 & 3 & Set & 1 & 2 & 3 & Set & 1 & 2 & 3 \\
\hline 1 & -1 & -1 & -1 & 3 & -1 & -1 & -1 & 5 & -1 & -1 & -1 \\
1 & -1 & +1 & +1 & 3 & +1 & -1 & +1 & 5 & +1 & +1 & -1 \\
\hline 2 & +1 & -1 & +1 & 4 & -1 & +1 & +1 & 6 & -1 & +1 & +1 \\
2 & +1 & +1 & -1 & 4 & +1 & +1 & -1 & 6 & +1 & -1 & +1 \\
\hline
\end{tabular}

Table A.2: Smallest optimal variance-balanced conjoint design with sets of $m=4$ profiles, $k_{c}=1$ constant attribute and $k_{v}=2$ non-constant attributes.

\begin{tabular}{|c|ccc||c|ccc||cccc|}
\hline & \multicolumn{3}{|c|}{ Attributes } & \multicolumn{4}{|c|}{} & \multicolumn{3}{|c||}{ Attributes } & \multicolumn{3}{|c|}{ Attributes } \\
Set & 1 & 2 & 3 & Set & 1 & 2 & 3 & Set & 1 & 2 & 3 \\
\hline 1 & -1 & -1 & -1 & 3 & -1 & -1 & -1 & 5 & -1 & -1 & -1 \\
1 & -1 & +1 & +1 & 3 & +1 & -1 & +1 & 5 & +1 & +1 & -1 \\
1 & -1 & -1 & +1 & 3 & -1 & -1 & +1 & 5 & -1 & +1 & -1 \\
1 & -1 & +1 & -1 & 3 & +1 & -1 & -1 & 5 & +1 & -1 & -1 \\
\hline 2 & +1 & -1 & -1 & 4 & -1 & +1 & -1 & 6 & -1 & -1 & +1 \\
2 & +1 & +1 & +1 & 4 & +1 & +1 & +1 & 6 & +1 & +1 & +1 \\
2 & +1 & -1 & +1 & 4 & -1 & +1 & +1 & 6 & -1 & +1 & +1 \\
2 & +1 & +1 & -1 & 4 & +1 & +1 & -1 & 6 & +1 & -1 & +1 \\
\hline
\end{tabular}


Table A.3: Smallest optimal variance-balanced conjoint design with sets of $m=2$ profiles, $k_{c}=1$ constant attribute and $k_{v}=3$ non-constant attributes.

\begin{tabular}{|c|cccc||c|cccc|}
\hline & \multicolumn{4}{|c|}{ Attributes } & \multicolumn{4}{|c|}{ Attributes } \\
Set & 1 & 2 & 3 & 4 & Set & 1 & 2 & 3 & 4 \\
\hline 1 & -1 & -1 & -1 & -1 & 9 & -1 & -1 & -1 & -1 \\
1 & -1 & +1 & +1 & +1 & 9 & +1 & +1 & -1 & +1 \\
\hline 2 & -1 & -1 & -1 & +1 & 10 & -1 & -1 & -1 & +1 \\
2 & -1 & +1 & +1 & -1 & 10 & +1 & +1 & -1 & -1 \\
\hline 3 & +1 & -1 & +1 & -1 & 11 & -1 & +1 & +1 & -1 \\
3 & +1 & +1 & -1 & +1 & 11 & +1 & -1 & +1 & +1 \\
\hline 4 & +1 & -1 & +1 & +1 & 12 & -1 & +1 & +1 & +1 \\
4 & +1 & +1 & -1 & -1 & 12 & +1 & -1 & +1 & -1 \\
\hline 5 & -1 & -1 & -1 & -1 & 13 & -1 & -1 & -1 & -1 \\
5 & +1 & -1 & +1 & +1 & 13 & +1 & +1 & +1 & -1 \\
\hline 6 & -1 & -1 & -1 & +1 & 14 & -1 & -1 & +1 & -1 \\
6 & +1 & -1 & +1 & -1 & 14 & +1 & +1 & -1 & -1 \\
\hline 7 & -1 & +1 & +1 & -1 & 15 & -1 & +1 & -1 & +1 \\
7 & +1 & +1 & -1 & +1 & 15 & +1 & -1 & +1 & +1 \\
\hline 8 & -1 & +1 & +1 & +1 & 16 & -1 & +1 & +1 & +1 \\
8 & +1 & +1 & -1 & -1 & 16 & +1 & -1 & -1 & +1 \\
\hline
\end{tabular}

Table A.4: Smallest optimal variance-balanced conjoint design with sets of $m=4$ profiles, $k_{c}=1$ constant attribute and $k_{v}=3$ non-constant attributes.

\begin{tabular}{|c|cccc||c|cccc|}
\hline & \multicolumn{4}{|c||}{ Attributes } & \multicolumn{4}{c|}{ Attributes } \\
Set & 1 & 2 & 3 & 4 & Set & 1 & 2 & 3 & 4 \\
\hline 1 & -1 & -1 & -1 & -1 & 5 & -1 & -1 & -1 & -1 \\
1 & -1 & +1 & +1 & +1 & 5 & +1 & +1 & -1 & +1 \\
1 & -1 & -1 & -1 & +1 & 5 & -1 & -1 & -1 & +1 \\
1 & -1 & +1 & +1 & -1 & 5 & +1 & +1 & -1 & -1 \\
\hline 2 & +1 & -1 & +1 & -1 & 6 & -1 & +1 & +1 & -1 \\
2 & +1 & +1 & -1 & +1 & 6 & +1 & -1 & +1 & +1 \\
2 & +1 & -1 & +1 & +1 & 6 & -1 & +1 & +1 & +1 \\
2 & +1 & +1 & -1 & -1 & 6 & +1 & -1 & +1 & -1 \\
\hline 3 & -1 & -1 & -1 & -1 & 7 & -1 & -1 & -1 & -1 \\
3 & +1 & -1 & +1 & +1 & 7 & +1 & +1 & +1 & -1 \\
3 & -1 & -1 & -1 & +1 & 7 & -1 & -1 & +1 & -1 \\
3 & +1 & -1 & +1 & -1 & 7 & +1 & +1 & -1 & -1 \\
\hline 4 & -1 & +1 & +1 & -1 & 8 & -1 & +1 & -1 & +1 \\
4 & +1 & +1 & -1 & +1 & 8 & +1 & -1 & +1 & +1 \\
4 & -1 & +1 & +1 & +1 & 8 & -1 & +1 & +1 & +1 \\
4 & +1 & +1 & -1 & -1 & 8 & +1 & -1 & -1 & +1 \\
\hline
\end{tabular}


Table A.5: Smallest optimal variance-balanced conjoint design with sets of $m=8$ profiles, $k_{c}=1$ constant attribute and $k_{v}=3$ non-constant attributes.

\begin{tabular}{|c|cccc||c|cccc|}
\hline & \multicolumn{3}{|c|}{ Attributes } & \multicolumn{4}{|c|}{ Attributes } \\
Set & 1 & 2 & 3 & 4 & Set & 1 & 2 & 3 & 4 \\
\hline 1 & -1 & -1 & -1 & -1 & 5 & -1 & -1 & -1 & -1 \\
1 & -1 & +1 & +1 & +1 & 5 & +1 & +1 & -1 & +1 \\
1 & -1 & -1 & -1 & +1 & 5 & -1 & -1 & -1 & +1 \\
1 & -1 & +1 & +1 & -1 & 5 & +1 & +1 & -1 & -1 \\
1 & -1 & -1 & +1 & -1 & 5 & -1 & +1 & -1 & -1 \\
1 & -1 & +1 & -1 & +1 & 5 & +1 & -1 & -1 & +1 \\
1 & -1 & -1 & +1 & +1 & 5 & -1 & +1 & -1 & +1 \\
1 & -1 & +1 & -1 & -1 & 5 & +1 & -1 & -1 & -1 \\
\hline 2 & +1 & -1 & -1 & -1 & 6 & -1 & -1 & +1 & -1 \\
2 & +1 & +1 & +1 & +1 & 6 & +1 & +1 & +1 & +1 \\
2 & +1 & -1 & -1 & +1 & 6 & -1 & -1 & +1 & +1 \\
2 & +1 & +1 & +1 & -1 & 6 & +1 & +1 & +1 & -1 \\
2 & +1 & -1 & +1 & -1 & 6 & -1 & +1 & +1 & -1 \\
2 & +1 & +1 & -1 & +1 & 6 & +1 & -1 & +1 & +1 \\
2 & +1 & -1 & +1 & +1 & 6 & -1 & +1 & +1 & +1 \\
2 & +1 & +1 & -1 & -1 & 6 & +1 & -1 & +1 & -1 \\
\hline 3 & -1 & -1 & -1 & -1 & 7 & -1 & -1 & -1 & -1 \\
3 & +1 & -1 & +1 & +1 & 7 & +1 & +1 & +1 & -1 \\
3 & -1 & -1 & -1 & +1 & 7 & -1 & -1 & +1 & -1 \\
3 & +1 & -1 & +1 & -1 & 7 & +1 & +1 & -1 & -1 \\
3 & -1 & +1 & +1 & -1 & 7 & -1 & +1 & -1 & -1 \\
3 & +1 & +1 & -1 & +1 & 7 & +1 & -1 & +1 & -1 \\
3 & -1 & +1 & +1 & +1 & 7 & -1 & +1 & +1 & -1 \\
3 & +1 & +1 & -1 & -1 & 7 & +1 & -1 & -1 & -1 \\
\hline 4 & -1 & -1 & -1 & -1 & 8 & -1 & -1 & -1 & +1 \\
4 & +1 & -1 & +1 & +1 & 8 & +1 & +1 & +1 & +1 \\
4 & -1 & -1 & -1 & +1 & 8 & -1 & -1 & +1 & +1 \\
4 & +1 & -1 & +1 & -1 & 8 & +1 & +1 & -1 & +1 \\
4 & -1 & +1 & +1 & -1 & 8 & -1 & +1 & -1 & +1 \\
4 & +1 & +1 & -1 & +1 & 8 & +1 & -1 & +1 & +1 \\
4 & -1 & +1 & +1 & +1 & 8 & -1 & +1 & +1 & +1 \\
4 & +1 & +1 & -1 & -1 & 8 & +1 & -1 & -1 & +1 \\
\hline
\end{tabular}


Table A.6: Smallest optimal variance-balanced conjoint design with sets of $m=2$ profiles, $k_{c}=1$ constant attribute and $k_{v}=4$ non-constant attributes.

\begin{tabular}{|c|ccccc||c|ccccc|}
\hline & \multicolumn{7}{|c|}{ Attributes } & \multicolumn{5}{|c|}{ Attributes } \\
Set & 1 & 2 & 3 & 4 & 5 & Set & 1 & 2 & 3 & 4 & 5 \\
\hline 1 & -1 & -1 & -1 & -1 & -1 & 11 & -1 & +1 & +1 & -1 & +1 \\
1 & -1 & +1 & +1 & +1 & +1 & 11 & +1 & -1 & +1 & +1 & -1 \\
\hline 2 & -1 & -1 & -1 & +1 & +1 & 12 & -1 & +1 & +1 & +1 & -1 \\
2 & -1 & +1 & +1 & -1 & -1 & 12 & +1 & -1 & +1 & -1 & +1 \\
\hline 3 & +1 & -1 & +1 & -1 & +1 & 13 & -1 & -1 & -1 & -1 & +1 \\
3 & +1 & +1 & -1 & +1 & -1 & 13 & +1 & +1 & +1 & -1 & -1 \\
\hline 4 & +1 & -1 & +1 & +1 & -1 & 14 & -1 & -1 & +1 & -1 & -1 \\
4 & +1 & +1 & -1 & -1 & +1 & 14 & +1 & +1 & -1 & -1 & +1 \\
\hline 5 & -1 & -1 & -1 & -1 & +1 & 15 & -1 & +1 & -1 & +1 & -1 \\
5 & +1 & -1 & +1 & +1 & -1 & 15 & +1 & -1 & +1 & +1 & +1 \\
\hline 6 & -1 & -1 & -1 & +1 & -1 & 16 & -1 & +1 & +1 & +1 & +1 \\
6 & +1 & -1 & +1 & -1 & +1 & 16 & +1 & -1 & -1 & +1 & -1 \\
\hline 7 & -1 & +1 & +1 & -1 & -1 & 17 & -1 & -1 & -1 & -1 & -1 \\
7 & +1 & +1 & -1 & +1 & +1 & 17 & +1 & +1 & +1 & +1 & -1 \\
\hline 8 & -1 & +1 & +1 & +1 & +1 & 18 & -1 & -1 & +1 & +1 & -1 \\
8 & +1 & +1 & -1 & -1 & -1 & 18 & +1 & +1 & -1 & -1 & -1 \\
\hline 9 & -1 & -1 & -1 & -1 & -1 & 19 & -1 & +1 & -1 & +1 & +1 \\
9 & +1 & +1 & -1 & +1 & +1 & 19 & +1 & -1 & +1 & -1 & +1 \\
\hline 10 & -1 & -1 & -1 & +1 & +1 & 20 & -1 & +1 & +1 & -1 & +1 \\
10 & +1 & +1 & -1 & -1 & -1 & 20 & +1 & -1 & -1 & +1 & +1 \\
\hline
\end{tabular}


Table A.7: Smallest optimal variance-balanced conjoint design with sets of $m=4$ profiles, $k_{c}=1$ constant attribute and $k_{v}=4$ non-constant attributes.

\begin{tabular}{|c|ccccc||c|ccccc|}
\hline & \multicolumn{9}{|c|}{ Attributes } & \multicolumn{5}{c|}{ Attributes } \\
Set & 1 & 2 & 3 & 4 & 5 & Set & 1 & 2 & 3 & 4 & 5 \\
\hline 1 & -1 & -1 & -1 & -1 & -1 & 6 & -1 & +1 & +1 & -1 & +1 \\
1 & -1 & +1 & +1 & +1 & +1 & 6 & +1 & -1 & +1 & +1 & -1 \\
1 & -1 & -1 & -1 & +1 & +1 & 6 & -1 & +1 & +1 & +1 & -1 \\
1 & -1 & +1 & +1 & -1 & -1 & 6 & +1 & -1 & +1 & -1 & +1 \\
\hline 2 & +1 & -1 & +1 & -1 & +1 & 7 & -1 & -1 & -1 & -1 & +1 \\
2 & +1 & +1 & -1 & +1 & -1 & 7 & +1 & +1 & +1 & -1 & -1 \\
2 & +1 & -1 & +1 & +1 & -1 & 7 & -1 & -1 & +1 & -1 & -1 \\
2 & +1 & +1 & -1 & -1 & +1 & 7 & +1 & +1 & -1 & -1 & +1 \\
\hline 3 & -1 & -1 & -1 & -1 & +1 & 8 & -1 & +1 & -1 & +1 & -1 \\
3 & +1 & -1 & +1 & +1 & -1 & 8 & +1 & -1 & +1 & +1 & +1 \\
3 & -1 & -1 & -1 & +1 & -1 & 8 & -1 & +1 & +1 & +1 & +1 \\
3 & +1 & -1 & +1 & -1 & +1 & 8 & +1 & -1 & -1 & +1 & -1 \\
\hline 4 & -1 & +1 & +1 & -1 & -1 & 9 & -1 & -1 & -1 & -1 & -1 \\
4 & +1 & +1 & -1 & +1 & +1 & 9 & +1 & +1 & +1 & +1 & -1 \\
4 & -1 & +1 & +1 & +1 & +1 & 9 & -1 & -1 & +1 & +1 & -1 \\
4 & +1 & +1 & -1 & -1 & -1 & 9 & +1 & +1 & -1 & -1 & -1 \\
\hline 5 & -1 & -1 & -1 & -1 & -1 & 10 & -1 & +1 & -1 & +1 & +1 \\
5 & +1 & +1 & -1 & +1 & +1 & 10 & +1 & -1 & +1 & -1 & +1 \\
5 & -1 & -1 & -1 & +1 & +1 & 10 & -1 & +1 & +1 & -1 & +1 \\
5 & +1 & +1 & -1 & -1 & -1 & 10 & +1 & -1 & -1 & +1 & +1 \\
\hline
\end{tabular}


Table A.8: Smallest optimal variance-balanced conjoint design with sets of $m=8$ profiles, $k_{c}=1$ constant attribute and $k_{v}=4$ non-constant attributes.

\begin{tabular}{|c|ccccc||c|ccccc|}
\hline & \multicolumn{7}{|c|}{ Attributes } & \multicolumn{7}{c|}{ Attributes } \\
Set & 1 & 2 & 3 & 4 & 5 & Set & 1 & 2 & 3 & 4 & 5 \\
\hline 1 & -1 & -1 & -1 & -1 & -1 & 6 & -1 & -1 & +1 & -1 & +1 \\
1 & -1 & +1 & +1 & +1 & +1 & 6 & +1 & +1 & +1 & +1 & -1 \\
1 & -1 & -1 & -1 & +1 & +1 & 6 & -1 & -1 & +1 & +1 & -1 \\
1 & -1 & +1 & +1 & -1 & -1 & 6 & +1 & +1 & +1 & -1 & +1 \\
1 & +1 & -1 & +1 & -1 & +1 & 6 & -1 & +1 & +1 & -1 & -1 \\
1 & +1 & +1 & -1 & +1 & -1 & 6 & +1 & -1 & +1 & +1 & +1 \\
1 & +1 & -1 & +1 & +1 & -1 & 6 & -1 & +1 & +1 & +1 & +1 \\
1 & +1 & +1 & -1 & -1 & +1 & 6 & +1 & -1 & +1 & -1 & -1 \\
\hline 2 & -1 & -1 & -1 & -1 & +1 & 7 & -1 & -1 & -1 & -1 & -1 \\
2 & -1 & +1 & +1 & +1 & -1 & 7 & +1 & +1 & +1 & -1 & +1 \\
2 & -1 & -1 & -1 & +1 & -1 & 7 & -1 & -1 & +1 & -1 & +1 \\
2 & -1 & +1 & +1 & -1 & +1 & 7 & +1 & +1 & -1 & -1 & -1 \\
2 & +1 & -1 & +1 & -1 & -1 & 7 & -1 & +1 & -1 & -1 & +1 \\
2 & +1 & +1 & -1 & +1 & +1 & 7 & +1 & -1 & +1 & -1 & -1 \\
2 & +1 & -1 & +1 & +1 & +1 & 7 & -1 & +1 & +1 & -1 & -1 \\
2 & +1 & +1 & -1 & -1 & -1 & 7 & +1 & -1 & -1 & -1 & +1 \\
\hline 3 & -1 & -1 & -1 & -1 & -1 & 8 & -1 & -1 & -1 & +1 & +1 \\
3 & +1 & -1 & +1 & +1 & +1 & 8 & +1 & +1 & +1 & +1 & -1 \\
3 & -1 & -1 & -1 & +1 & +1 & 8 & -1 & -1 & +1 & +1 & -1 \\
3 & +1 & -1 & +1 & -1 & -1 & 8 & +1 & +1 & -1 & +1 & +1 \\
3 & -1 & +1 & +1 & -1 & +1 & 8 & -1 & +1 & -1 & +1 & -1 \\
3 & +1 & +1 & -1 & +1 & -1 & 8 & +1 & -1 & +1 & +1 & +1 \\
3 & -1 & +1 & +1 & +1 & -1 & 8 & -1 & +1 & +1 & +1 & +1 \\
3 & +1 & +1 & -1 & -1 & +1 & 8 & +1 & -1 & -1 & +1 & -1 \\
\hline 4 & -1 & -1 & -1 & -1 & +1 & 9 & -1 & -1 & -1 & -1 & -1 \\
4 & +1 & -1 & +1 & +1 & -1 & 9 & +1 & +1 & +1 & +1 & -1 \\
4 & -1 & -1 & -1 & +1 & -1 & 9 & -1 & -1 & +1 & +1 & -1 \\
4 & +1 & -1 & +1 & -1 & +1 & 9 & +1 & +1 & -1 & -1 & -1 \\
4 & -1 & +1 & +1 & -1 & -1 & 9 & -1 & +1 & -1 & +1 & -1 \\
4 & +1 & +1 & -1 & +1 & +1 & 9 & +1 & -1 & +1 & -1 & -1 \\
4 & -1 & +1 & +1 & +1 & +1 & 9 & -1 & +1 & +1 & -1 & -1 \\
4 & +1 & +1 & -1 & -1 & -1 & 9 & +1 & -1 & -1 & +1 & -1 \\
\hline 5 & -1 & -1 & -1 & -1 & -1 & 10 & -1 & -1 & -1 & +1 & +1 \\
5 & +1 & +1 & -1 & +1 & +1 & 10 & +1 & +1 & +1 & -1 & +1 \\
5 & -1 & -1 & -1 & +1 & +1 & 10 & -1 & -1 & +1 & -1 & +1 \\
5 & +1 & +1 & -1 & -1 & -1 & 10 & +1 & +1 & -1 & +1 & +1 \\
5 & -1 & +1 & -1 & -1 & +1 & 10 & -1 & +1 & -1 & -1 & +1 \\
5 & +1 & -1 & -1 & +1 & -1 & 10 & +1 & -1 & +1 & +1 & +1 \\
5 & -1 & +1 & -1 & +1 & -1 & 10 & -1 & +1 & +1 & +1 & +1 \\
5 & +1 & -1 & -1 & -1 & +1 & 10 & +1 & -1 & -1 & -1 & +1 \\
\hline
\end{tabular}




\section{A.2. Designs with more than one constant attribute: $k_{c}>1$}

Table A.9: Smallest optimal variance-balanced conjoint design with sets of $m=2$ profiles, $k_{c}=2$ constant attributes and $k_{v}=4$ non-constant attributes.

\begin{tabular}{|c|c|c|c|c|c|c|c|c|c|c|c|c|c|}
\hline \multirow{3}{*}{ 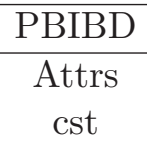 } & \multirow[b]{3}{*}{ Set } & \multicolumn{6}{|c|}{ Constant attribute design } & \multicolumn{6}{|c|}{ Conjoint design } \\
\hline & & \multirow[b]{2}{*}{1} & \multicolumn{4}{|c|}{ Attributes } & \multirow[b]{2}{*}{6} & \multicolumn{6}{|c|}{ Attributes } \\
\hline & & & 2 & 3 & 4 & 5 & & 1 & 2 & 3 & 4 & 5 & 6 \\
\hline & 1 & -1 & - & - & -1 & - & - & -1 & -1 & -1 & -1 & -1 & -1 \\
\hline & 1 & -1 & - & - & -1 & - & - & -1 & +1 & +1 & -1 & +1 & +1 \\
\hline & 2 & -1 & - & - & +1 & - & - & -1 & -1 & -1 & +1 & +1 & +1 \\
\hline & 2 & -1 & - & - & †1 & - & - & -1 & +1 & +1 & +1 & -1 & -1 \\
\hline & 3 & & & & & - & & +1 & -1 & +1 & -1 & -1 & +1 \\
\hline & 3 & +1 & - & - & -1 & - & - & +1 & +1 & -1 & -1 & +1 & -1 \\
\hline & 4 & +1 & - & - & +1 & - & $\overline{-}$ & +1 & -1 & +1 & +1 & +1 & -1 \\
\hline & 4 & +1 & - & - & +1 & - & - & +1 & +1 & -1 & +1 & -1 & +1 \\
\hline \multirow{8}{*}{2} & 5 & $\overline{-}$ & & - & & & & -1 & -1 & -1 & -1 & -1 & +1 \\
\hline & 5 & & -1 & & & -1 & & +1 & -1 & +1 & +1 & -1 & -1 \\
\hline & 6 & - & -1 & - & - & +1 & - & -1 & -1 & -1 & +1 & +1 & -1 \\
\hline & 6 & & & & & & & +1 & -1 & +1 & -1 & +1 & +1 \\
\hline & 7 & - & +1 &  & - & & & -1 & +1 & +1 & -1 & -1 & -1 \\
\hline & 7 & - & +1 & - & - & -1 & & +1 & +1 & -1 & +1 & -1 & +1 \\
\hline & 8 & & +1 & & & & & -1 & +1 & +1 & +1 & +1 & +1 \\
\hline & 8 & - & +1 & & - & +1 & & +1 & +1 & -1 & -1 & +1 & -1 \\
\hline \multirow{8}{*}{$\begin{array}{ll}3 & 6\end{array}$} & 9 & - & 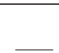 & -1 & - & & -1 & -1 & -1 & -1 & -1 & -1 & -1 \\
\hline & 9 & - & - & 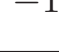 & & & & +1 & +1 & -1 & +1 & +1 & -1 \\
\hline & 10 & $\overline{-}$ & $\overline{-}$ & -1 & - & & & -1 & -1 & -1 & +1 & +1 & +1 \\
\hline & 10 & - & - & -1 & - & - & +1 & +1 & +1 & -1 & -1 & -1 & +1 \\
\hline & 11 & $\bar{L}$ & - & +1 & & & & -1 & +1 & +1 & -1 & +1 & -1 \\
\hline & 11 & - & - & +1 & - & - & & +1 & -1 & +1 & +1 & -1 & -1 \\
\hline & 12 & $\overline{-}$ & $\overline{-}$ & +1 & $\overline{-}$ & - & 1 & -1 & +1 & +1 & +1 & -1 & +1 \\
\hline & 12 & - & - & +1 & - & - & +1 & +1 & -1 & +1 & -1 & +1 & +1 \\
\hline
\end{tabular}


Table A.10: Smallest optimal variance-balanced conjoint design with sets of $m=4$ profiles, $k_{c}=2$ constant attributes and $k_{v}=4$ non-constant attributes.

\begin{tabular}{|c|c|c|c|c|c|c|c|c|c|c|c|c|c|}
\hline \multirow{3}{*}{ 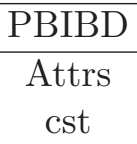 } & \multirow[b]{3}{*}{ Set } & \multicolumn{6}{|c|}{ Constant attribute design } & \multicolumn{6}{|c|}{ Conjoint design } \\
\hline & & & & Attr & butes & & & & & Attri & outes & & \\
\hline & & 1 & 2 & 3 & 4 & 5 & 6 & 1 & 2 & 3 & 4 & 5 & 6 \\
\hline & 1 & \multirow{4}{*}{-1} & \multirow{4}{*}{-} & \multirow{4}{*}{ - } & \multirow{4}{*}{-1} & \multirow{4}{*}{ - } & \multirow{4}{*}{ - } & -1 & -1 & -1 & -1 & -1 & -1 \\
\hline & 1 & & & & & & & -1 & +1 & +1 & -1 & +1 & +1 \\
\hline & 1 & & & & & & & -1 & -1 & -1 & -1 & +1 & +1 \\
\hline & 1 & & & & & & & -1 & +1 & +1 & -1 & -1 & -1 \\
\hline & 2 & \multirow{4}{*}{-1} & \multirow{4}{*}{ - } & \multirow{4}{*}{ - } & \multirow{4}{*}{+1} & \multirow{4}{*}{ - } & \multirow{4}{*}{ - } & -1 & -1 & +1 & +1 & -1 & +1 \\
\hline & 2 & & & & & & & -1 & +1 & -1 & +1 & +1 & -1 \\
\hline & 2 & & & & & & & -1 & -1 & +1 & +1 & +1 & -1 \\
\hline & 2 & & & & & & & -1 & +1 & -1 & +1 & -1 & +1 \\
\hline & 3 & \multirow{4}{*}{+1} & \multirow{4}{*}{ - } & \multirow{4}{*}{ — } & \multirow{4}{*}{-1} & \multirow{4}{*}{ - } & \multirow{4}{*}{ - } & +1 & -1 & -1 & -1 & -1 & +1 \\
\hline & 3 & & & & & & & +1 & +1 & +1 & -1 & +1 & -1 \\
\hline & 3 & & & & & & & +1 & -1 & -1 & -1 & +1 & -1 \\
\hline & 3 & & & & & & & +1 & +1 & +1 & -1 & -1 & +1 \\
\hline & 4 & & & & & & & +1 & -1 & +1 & +1 & -1 & -1 \\
\hline & 4 & +1 & & & +1 & & & +1 & +1 & -1 & +1 & +1 & +1 \\
\hline & 4 & +1 & - & 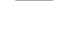 & +1 & 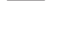 & 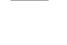 & +1 & -1 & +1 & +1 & +1 & +1 \\
\hline 4 & 4 & & & & & & & +1 & +1 & -1 & +1 & -1 & -1 \\
\hline$\vdots$ & $\vdots$ & & & & & & & & & & & & \\
\hline & 9 & & & & & & & -1 & -1 & -1 & -1 & -1 & -1 \\
\hline & 9 & & & -1 & & & 1 & +1 & +1 & -1 & +1 & +1 & -1 \\
\hline & 9 & - & - & -1 & & - & -1 & -1 & -1 & -1 & +1 & +1 & -1 \\
\hline & 9 & & & & & & & +1 & +1 & -1 & -1 & -1 & -1 \\
\hline & 10 & & & & & & & -1 & +1 & -1 & -1 & +1 & +1 \\
\hline & 10 & & & 1 & & & 1 & +1 & -1 & -1 & +1 & -1 & +1 \\
\hline & 10 & - & - & -1 & - & - & +1 & -1 & +1 & -1 & +1 & -1 & +1 \\
\hline & 10 & & & & & & & +1 & -1 & -1 & -1 & +1 & +1 \\
\hline & 11 & & & & & & & -1 & -1 & +1 & -1 & +1 & -1 \\
\hline & 11 & & & & & & & +1 & +1 & +1 & +1 & -1 & -1 \\
\hline & 11 & - & - & +1 & - & - & -1 & -1 & -1 & +1 & +1 & -1 & -1 \\
\hline & 11 & & & & & & & +1 & +1 & +1 & -1 & +1 & -1 \\
\hline & 12 & & & & & & & -1 & +1 & +1 & -1 & -1 & +1 \\
\hline & 12 & - & $\overline{-}$ & +1 & $\overline{-}$ & $\overline{-}$ & +1 & +1 & -1 & +1 & +1 & +1 & +1 \\
\hline & 12 & & - & +1 & - & - & +1 & -1 & +1 & +1 & +1 & +1 & +1 \\
\hline & 12 & & & & & & & +1 & -1 & +1 & -1 & -1 & +1 \\
\hline
\end{tabular}


Table A.11: Smallest optimal variance-balanced conjoint design with sets of $m=8$ profiles, $k_{c}=2$ constant attributes and $k_{v}=4$ non-constant attributes.

\begin{tabular}{|c|c|c|c|c|c|c|c|c|c|c|c|c|c|}
\hline \multirow{3}{*}{ 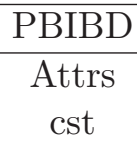 } & \multirow[b]{3}{*}{ Set } & \multicolumn{6}{|c|}{ Constant attribute design } & \multicolumn{6}{|c|}{ Conjoint design } \\
\hline & & \multicolumn{6}{|c|}{ Attributes } & \multicolumn{6}{|c|}{ Attributes } \\
\hline & & 1 & 2 & 3 & 4 & 5 & 6 & 1 & 2 & 3 & 4 & 5 & 6 \\
\hline \multirow{32}{*}{14} & 1 & \multirow{8}{*}{-1} & \multirow{8}{*}{ — } & \multirow{8}{*}{ - } & \multirow{8}{*}{-1} & \multirow{8}{*}{ — } & \multirow{8}{*}{ - } & -1 & -1 & -1 & -1 & -1 & -1 \\
\hline & 1 & & & & & & & -1 & +1 & +1 & -1 & +1 & +1 \\
\hline & 1 & & & & & & & -1 & -1 & -1 & -1 & +1 & +1 \\
\hline & 1 & & & & & & & -1 & +1 & +1 & -1 & -1 & -1 \\
\hline & 1 & & & & & & & -1 & -1 & +1 & -1 & -1 & +1 \\
\hline & 1 & & & & & & & -1 & +1 & -1 & -1 & +1 & -1 \\
\hline & 1 & & & & & & & -1 & -1 & +1 & -1 & +1 & -1 \\
\hline & 1 & & & & & & & -1 & +1 & -1 & -1 & -1 & +1 \\
\hline & 2 & \multirow{8}{*}{-1} & \multirow{8}{*}{ - } & \multirow{8}{*}{ - } & \multirow{8}{*}{+1} & \multirow{8}{*}{ — } & \multirow{8}{*}{ - } & -1 & -1 & -1 & +1 & -1 & +1 \\
\hline & 2 & & & & & & & -1 & +1 & +1 & +1 & +1 & -1 \\
\hline & 2 & & & & & & & -1 & -1 & -1 & +1 & +1 & -1 \\
\hline & 2 & & & & & & & -1 & +1 & +1 & +1 & -1 & +1 \\
\hline & 2 & & & & & & & -1 & -1 & +1 & +1 & -1 & -1 \\
\hline & 2 & & & & & & & -1 & +1 & -1 & +1 & +1 & +1 \\
\hline & 2 & & & & & & & -1 & -1 & +1 & +1 & +1 & +1 \\
\hline & 2 & & & & & & & -1 & +1 & -1 & +1 & -1 & -1 \\
\hline & 3 & \multirow{8}{*}{+1} & \multirow{8}{*}{ - } & \multirow{8}{*}{ - } & \multirow{8}{*}{-1} & \multirow{8}{*}{ - } & & +1 & -1 & -1 & -1 & -1 & -1 \\
\hline & 3 & & & & & & & +1 & +1 & +1 & -1 & +1 & +1 \\
\hline & 3 & & & & & & & +1 & -1 & -1 & -1 & +1 & +1 \\
\hline & 3 & & & & & & & +1 & +1 & +1 & -1 & -1 & -1 \\
\hline & 3 & & & & & & - & +1 & -1 & +1 & -1 & -1 & +1 \\
\hline & 3 & & & & & & & +1 & +1 & -1 & -1 & +1 & -1 \\
\hline & 3 & & & & & & & +1 & -1 & +1 & -1 & +1 & -1 \\
\hline & 3 & & & & & & & +1 & +1 & -1 & -1 & -1 & +1 \\
\hline & 4 & & & & & & & +1 & -1 & -1 & +1 & -1 & +1 \\
\hline & 4 & & & & & & & +1 & +1 & +1 & +1 & +1 & -1 \\
\hline & 4 & & & & & & & +1 & -1 & -1 & +1 & +1 & -1 \\
\hline & 4 & +1 & & 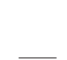 & +1 & & & +1 & +1 & +1 & +1 & -1 & +1 \\
\hline & 4 & +1 & - & - & +1 & - & & +1 & -1 & +1 & +1 & -1 & -1 \\
\hline & 4 & & & & & & & +1 & +1 & -1 & +1 & +1 & +1 \\
\hline & 4 & & & & & & & +1 & -1 & +1 & +1 & +1 & +1 \\
\hline & 4 & & & & & & & +1 & +1 & -1 & +1 & -1 & -1 \\
\hline$\vdots$ & $\vdots$ & & & & & & & & & & & & \\
\hline
\end{tabular}


continued from previous page

\begin{tabular}{|c|c|c|c|c|c|c|c|c|c|c|c|c|c|}
\hline \multirow{3}{*}{ 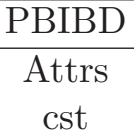 } & \multirow[b]{3}{*}{ Set } & \multicolumn{6}{|c|}{ Constant attribute design } & \multicolumn{6}{|c|}{ Conjoint design } \\
\hline & & \multicolumn{6}{|c|}{ Attributes } & \multicolumn{6}{|c|}{ Attributes } \\
\hline & & 1 & 2 & 3 & 4 & 5 & 6 & 1 & 2 & 3 & 4 & 5 & 6 \\
\hline & 9 & \multirow{8}{*}{-} & \multirow{8}{*}{ - } & \multirow{8}{*}{-1} & \multirow{8}{*}{ - } & \multirow{8}{*}{ - } & \multirow{8}{*}{-1} & -1 & -1 & -1 & -1 & -1 & -1 \\
\hline & 9 & & & & & & & +1 & +1 & -1 & +1 & +1 & -1 \\
\hline & 9 & & & & & & & -1 & -1 & -1 & +1 & +1 & -1 \\
\hline & 9 & & & & & & & +1 & +1 & -1 & -1 & -1 & -1 \\
\hline & 9 & & & & & & & -1 & +1 & -1 & -1 & +1 & -1 \\
\hline & 9 & & & & & & & +1 & -1 & -1 & +1 & -1 & -1 \\
\hline & 9 & & & & & & & -1 & +1 & -1 & +1 & -1 & -1 \\
\hline & 9 & & & & & & & +1 & -1 & -1 & -1 & +1 & -1 \\
\hline & 10 & \multirow{8}{*}{-} & \multirow{8}{*}{ - } & \multirow{8}{*}{-1} & \multirow{8}{*}{-} & \multirow{8}{*}{ - } & \multirow{8}{*}{+1} & -1 & -1 & -1 & -1 & +1 & +1 \\
\hline & 10 & & & & & & & +1 & +1 & -1 & +1 & -1 & +1 \\
\hline & 10 & & & & & & & -1 & -1 & -1 & +1 & -1 & +1 \\
\hline & 10 & & & & & & & +1 & +1 & -1 & -1 & +1 & +1 \\
\hline & 10 & & & & & & & -1 & +1 & -1 & -1 & -1 & +1 \\
\hline & 10 & & & & & & & +1 & -1 & -1 & +1 & +1 & +1 \\
\hline & 10 & & & & & & & -1 & +1 & -1 & +1 & +1 & +1 \\
\hline & 10 & & & & & & & +1 & -1 & -1 & -1 & -1 & +1 \\
\hline & 11 & \multirow{8}{*}{-} & \multirow{8}{*}{ - } & \multirow{8}{*}{+1} & \multirow{8}{*}{ - } & \multirow{8}{*}{-} & \multirow{8}{*}{-1} & -1 & -1 & +1 & -1 & -1 & -1 \\
\hline & 11 & & & & & & & +1 & +1 & +1 & +1 & +1 & -1 \\
\hline & 11 & & & & & & & -1 & -1 & +1 & +1 & +1 & -1 \\
\hline & 11 & & & & & & & +1 & +1 & +1 & -1 & -1 & -1 \\
\hline & 11 & & & & & & & -1 & +1 & +1 & -1 & +1 & -1 \\
\hline & 11 & & & & & & & +1 & -1 & +1 & +1 & -1 & -1 \\
\hline & 11 & & & & & & & -1 & +1 & +1 & +1 & -1 & -1 \\
\hline & 11 & & & & & & & +1 & -1 & +1 & -1 & +1 & -1 \\
\hline & 12 & & & & & & & -1 & -1 & +1 & -1 & +1 & +1 \\
\hline & 12 & & & & & & & +1 & +1 & +1 & +1 & -1 & +1 \\
\hline & 12 & & & & & & & -1 & -1 & +1 & +1 & -1 & +1 \\
\hline & 12 & 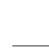 & & +1 & & & +1 & +1 & +1 & +1 & -1 & +1 & +1 \\
\hline & 12 &  & - & +1 & . & - & $T^{\prime} 1$ & -1 & +1 & +1 & -1 & -1 & +1 \\
\hline & 12 & & & & & & & +1 & -1 & +1 & +1 & +1 & +1 \\
\hline & 12 & & & & & & & -1 & +1 & +1 & +1 & +1 & +1 \\
\hline & 12 & & & & & & & +1 & -1 & +1 & -1 & -1 & +1 \\
\hline
\end{tabular}


Table A.12: Smallest optimal variance-balanced conjoint design with sets of $m=2$ profiles, $k_{c}=2$ constant attributes and $k_{v}=2$ non-constant attributes.

\begin{tabular}{|c|c|c|c|c|c|c|c|c|c|c|}
\hline \multirow{3}{*}{\multicolumn{2}{|c|}{ 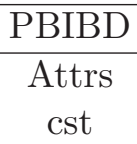 }} & \multirow[b]{3}{*}{ Set } & \multicolumn{4}{|c|}{ Cst attribute design } & \multicolumn{4}{|c|}{ Conjoint design } \\
\hline & & & \multicolumn{4}{|c|}{ Attributes } & \multicolumn{4}{|c|}{ Attributes } \\
\hline & & & 1 & 2 & 3 & 4 & 1 & 2 & 3 & 4 \\
\hline \multirow{4}{*}{1} & \multirow{4}{*}{2} & 1 & \multirow{2}{*}{-1} & \multirow{2}{*}{-1} & \multirow[t]{2}{*}{ - } & \multirow{2}{*}{ - } & -1 & -1 & -1 & -1 \\
\hline & & 1 & & & & & -1 & -1 & +1 & +1 \\
\hline & & 2 & \multirow{2}{*}{-1} & \multirow{2}{*}{+1} & \multirow{2}{*}{ - } & \multirow{2}{*}{ - } & -1 & +1 & -1 & +1 \\
\hline & & 2 & & & & & -1 & +1 & +1 & -1 \\
\hline \multirow{4}{*}{1} & \multirow{4}{*}{4} & 3 & \multirow{2}{*}{+1} & \multirow{2}{*}{-} & \multirow{2}{*}{ - } & \multirow{2}{*}{-1} & +1 & -1 & -1 & -1 \\
\hline & & 3 & & & & & +1 & +1 & +1 & -1 \\
\hline & & 4 & \multirow{2}{*}{+1} & \multirow{2}{*}{ - } & \multirow{2}{*}{ - } & \multirow{2}{*}{+1} & +1 & -1 & +1 & +1 \\
\hline & & 4 & & & & & +1 & +1 & -1 & +1 \\
\hline \multirow{4}{*}{2} & \multirow{4}{*}{3} & 5 & \multirow{2}{*}{ - } & \multirow{2}{*}{-1} & \multirow{2}{*}{-1} & \multirow{2}{*}{ - } & -1 & -1 & -1 & -1 \\
\hline & & 5 & & & & & +1 & -1 & -1 & +1 \\
\hline & & 6 & \multirow{2}{*}{ - } & \multirow{2}{*}{+1} & -1 & & -1 & +1 & -1 & +1 \\
\hline & & 6 & & & & & +1 & +1 & -1 & -1 \\
\hline & & 7 & & & & & -1 & -1 & +1 & -1 \\
\hline & & 7 & & & +1 & & +1 & +1 & +1 & -1 \\
\hline & & 8 & - & - & +1 & +1 & -1 & +1 & +1 & +1 \\
\hline & & 8 & & & & & +1 & -1 & +1 & +1 \\
\hline
\end{tabular}


Table A.13: Smallest optimal variance-balanced conjoint design with sets of $m=4$ profiles, $k_{c}=2$ constant attributes and $k_{v}=2$ non-constant attributes.

\begin{tabular}{|c|c|c|c|c|c|c|c|c|c|}
\hline \multirow{3}{*}{$\begin{array}{c}\text { PBIBD } \\
\text { Attrs } \\
\text { cst }\end{array}$} & \multirow[b]{3}{*}{ Set } & \multicolumn{4}{|c|}{ Cst attribute design } & \multicolumn{4}{|c|}{ Conjoint design } \\
\hline & & & Attr & bute & & & Attr & utes & \\
\hline & & 1 & 2 & 3 & 4 & 1 & 2 & 3 & 4 \\
\hline \multirow{8}{*}{2} & 1 & \multirow{4}{*}{-1} & \multirow{4}{*}{-1} & \multirow{4}{*}{$\ldots$} & \multirow{4}{*}{ - } & -1 & -1 & -1 & -1 \\
\hline & 1 & & & & & -1 & -1 & +1 & +1 \\
\hline & 1 & & & & & -1 & -1 & -1 & +1 \\
\hline & 1 & & & & & -1 & -1 & +1 & -1 \\
\hline & 2 & \multirow{4}{*}{-1} & \multirow{4}{*}{+1} & \multirow{4}{*}{ - } & \multirow{4}{*}{ - } & -1 & +1 & -1 & -1 \\
\hline & 2 & & & & & -1 & +1 & +1 & +1 \\
\hline & 2 & & & & & -1 & +1 & -1 & +1 \\
\hline & 2 & & & & & -1 & +1 & +1 & -1 \\
\hline \multirow{8}{*}{1} & 3 & \multirow{4}{*}{+1} & \multirow{4}{*}{ - } & \multirow{4}{*}{ - } & \multirow{4}{*}{-1} & +1 & -1 & -1 & -1 \\
\hline & 3 & & & & & +1 & +1 & +1 & -1 \\
\hline & 3 & & & & & +1 & -1 & +1 & -1 \\
\hline & 3 & & & & & +1 & +1 & -1 & -1 \\
\hline & 4 & \multirow{4}{*}{+1} & \multirow{4}{*}{ - } & \multirow{4}{*}{-} & \multirow{4}{*}{+1} & +1 & -1 & -1 & +1 \\
\hline & 4 & & & & & +1 & +1 & +1 & +1 \\
\hline & 4 & & & & & +1 & -1 & +1 & +1 \\
\hline & 4 & & & & & +1 & +1 & -1 & +1 \\
\hline \multirow{7}{*}{2} & 5 & \multirow{4}{*}{ - } & & & & -1 & -1 & -1 & -1 \\
\hline & 5 & & & & & +1 & -1 & -1 & +1 \\
\hline & 5 & & -1 & -1 & & -1 & -1 & -1 & +1 \\
\hline & 5 & & & & & +1 & -1 & -1 & -1 \\
\hline & 6 & & & & & -1 & +1 & -1 & -1 \\
\hline & 6 & & & & & +1 & +1 & -1 & +1 \\
\hline & 6 & - & +1 & -1 & & -1 & +1 & -1 & +1 \\
\hline & 6 & & & & & +1 & +1 & +1 & -1 \\
\hline & 7 & & & & & -1 & -1 & +1 & -1 \\
\hline & 7 & 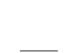 & & & & +1 & +1 & +1 & -1 \\
\hline & 7 & - & - & +1 & -1 & -1 & +1 & +1 & -1 \\
\hline 3 & 7 & & & & & +1 & -1 & +1 & -1 \\
\hline & 8 & & & & & -1 & -1 & +1 & +1 \\
\hline & 8 & - & $=$ & +1 & & +1 & +1 & +1 & +1 \\
\hline & 8 & - & - & +1 & +1 & -1 & +1 & +1 & +1 \\
\hline & 8 & & & & & +1 & -1 & +1 & +1 \\
\hline
\end{tabular}


Table A.14: Smallest optimal variance-balanced conjoint design with sets of $m=2$ profiles, $k_{c}=3$ constant attributes and $k_{v}=3$ non-constant attributes.

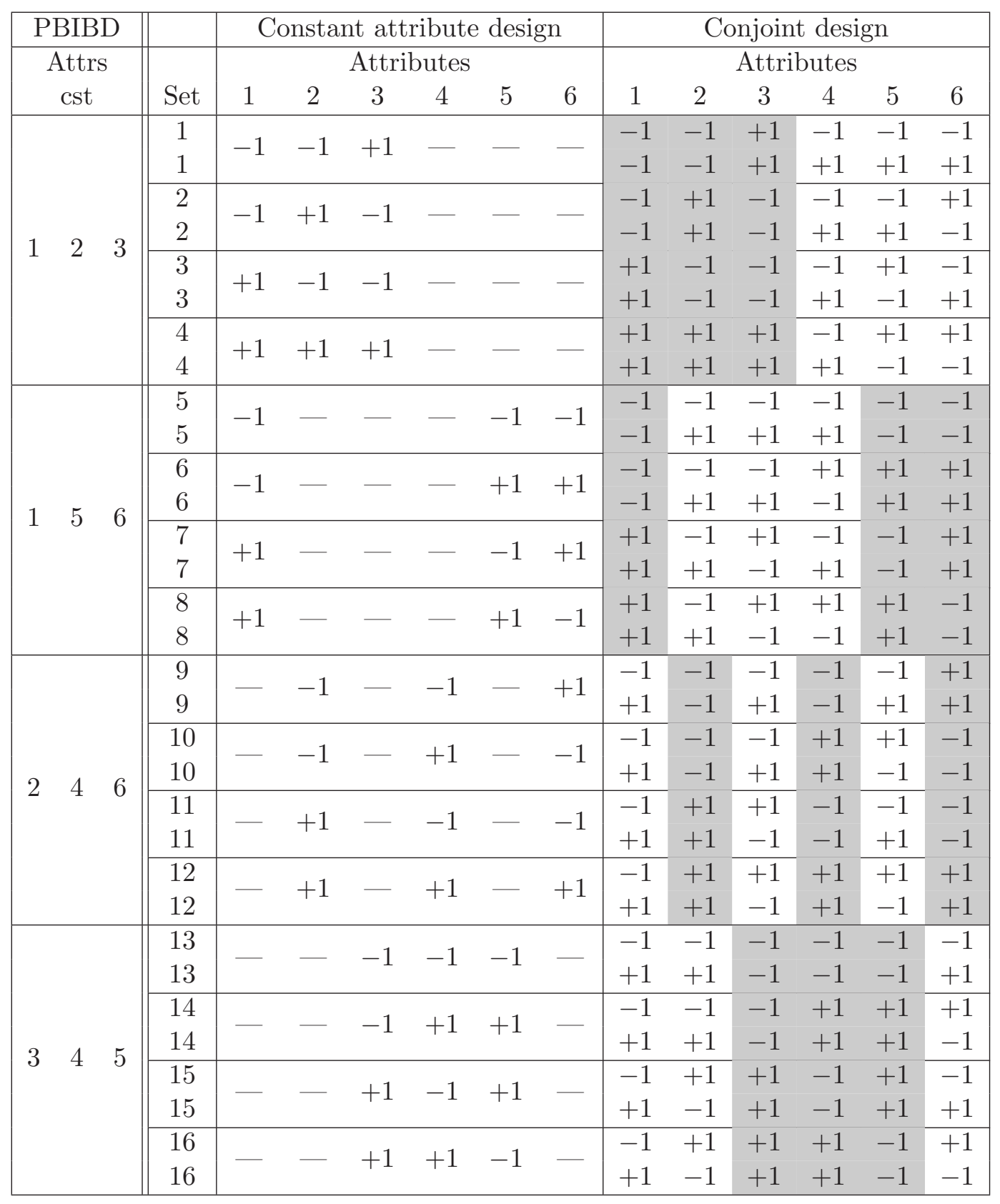




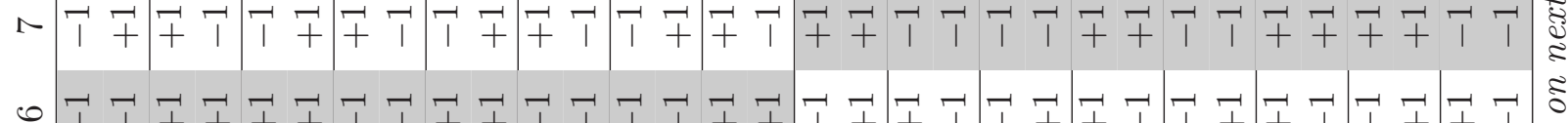
$+\sqrt{2}$ サろ

๓ ง

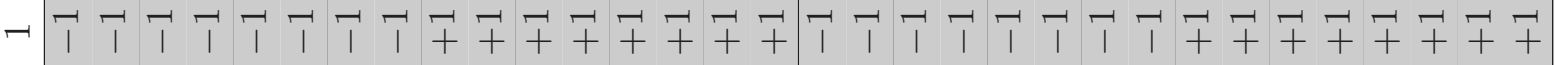

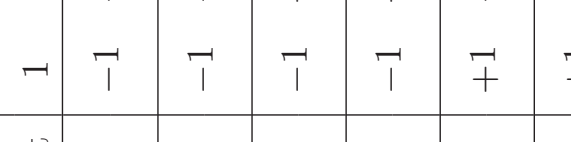

\section{(}

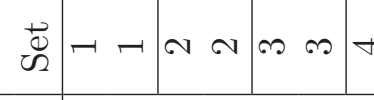

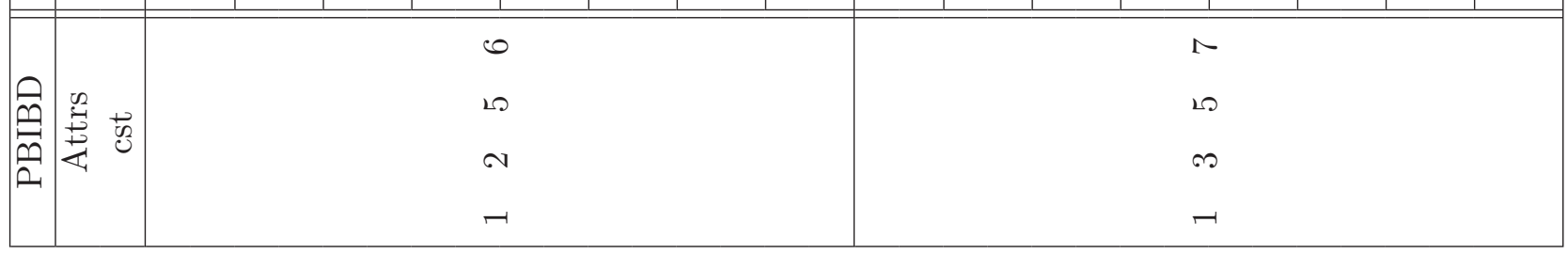




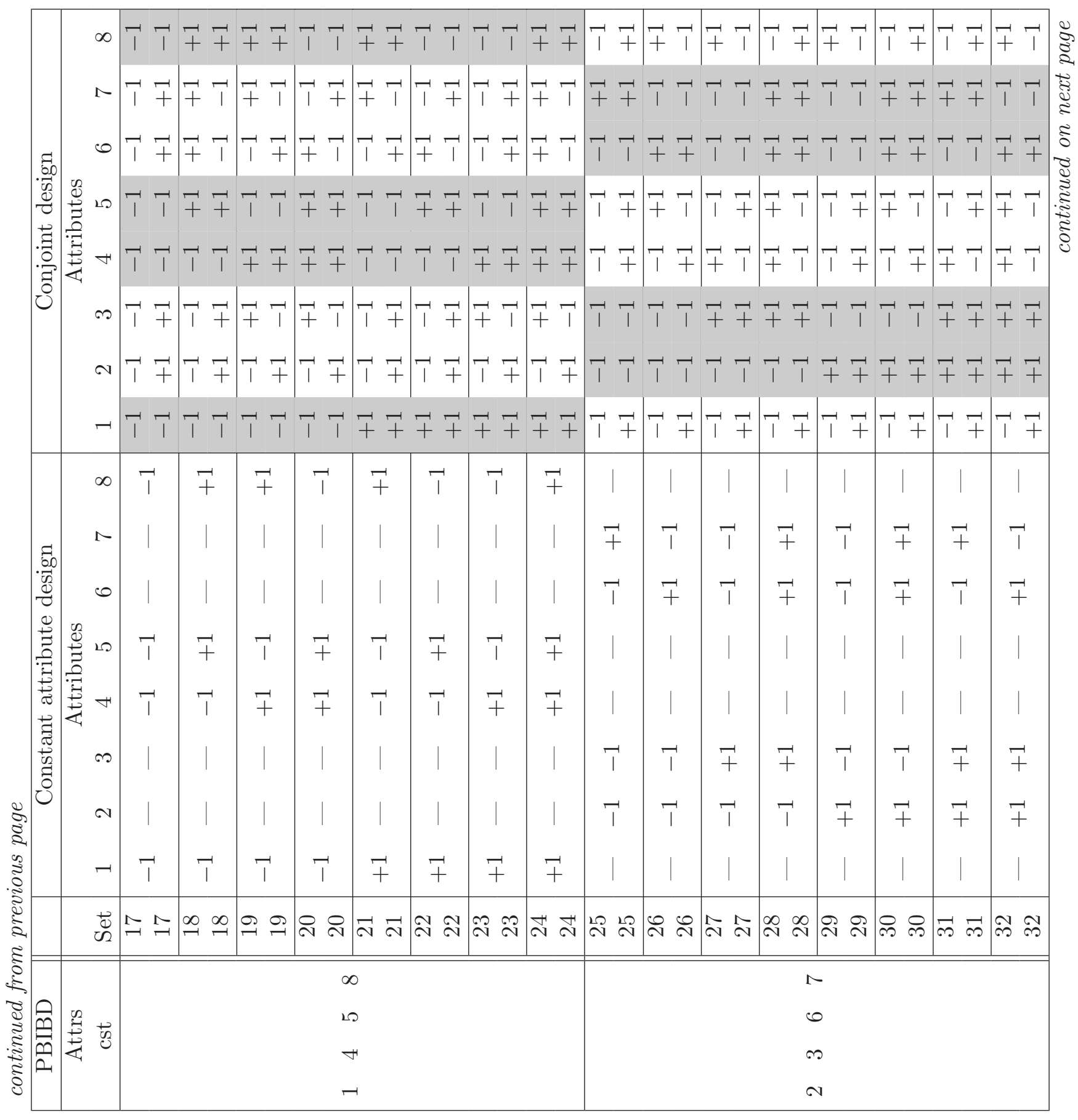




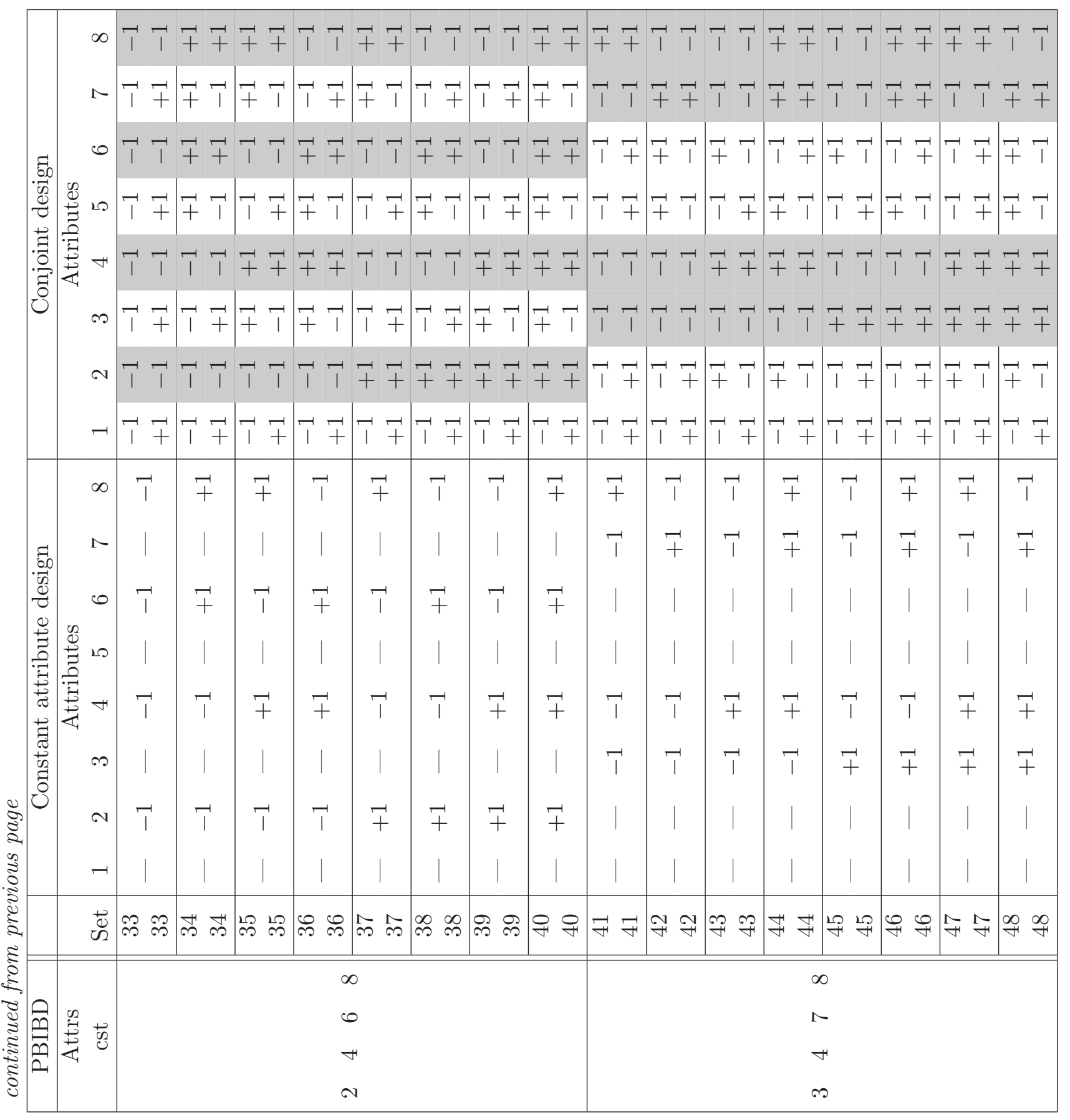




\section{Appendix B. Derivation of the information matrix}

In this appendix, we derive the information matrix of the optimal variance-balanced conjoint design in Table A.12 of Appendix A.2. Based on this example, we provide a general method to compute the information matrix of any optimal variance-balanced conjoint design.

The conjoint design in Table A.12 has $b=8$ sets of $m=2$ profiles, $k_{c}=2$ constant attributes and $k_{v}=2$ non-constant attributes. It is built on a PBIBD that identifies four combinations of constant attributes. Each attribute is constant in four profile sets. To compute the information matrix of the design, we go back to the work of Goos and Vandebroek (2001) which provides the basis for the information matrix (8). Under assumptions (3), (4) and (5), $\mathbf{V}$ is block diagonal, so that

$$
\mathbf{X}^{\prime} \mathbf{V}^{-1} \mathbf{X}=\sum_{i=1}^{b} \mathbf{X}_{i}^{\prime} \mathbf{V}_{m}^{-1} \mathbf{X}_{i}
$$

where

$$
\mathbf{V}_{m}^{-1}=\sigma_{\boldsymbol{\varepsilon}}^{-2}\left(\mathbf{I}_{m}-\frac{\rho}{1+\rho(m-1)} \mathbf{1}_{m} \mathbf{1}_{m}^{\prime}\right)
$$

According to (B.1), the information matrix of the design in Table A.12 is the sum of $b=8$ information matrices, one for each of the profile sets. We now compute the information matrix for each profile set and sum the matrices to obtain the total information matrix of the design. The design has profile sets of size $m=2$ so that

$$
\mathbf{V}_{2}^{-1}=\sigma_{\varepsilon}^{-2}\left(\mathbf{I}_{2}-\frac{\rho}{1+\rho} \mathbf{1}_{2} \mathbf{1}_{2}^{\prime}\right)
$$

To derive the information matrices of the individual profile sets, we write each profile set in terms of its constant and non-constant attributes. Therefore, we denote the levels of the two constant attributes in profile set $i$ by $w_{i 1} \mathbf{1}_{2}$ and $w_{i 2} \mathbf{1}_{2}$, where $w_{i 1}$ and $w_{i 2}$ equal either -1 and +1 , and we denote the levels of the two non-constant attributes by the two-dimensional vectors $\mathbf{s}_{i 1}$ and $\mathbf{s}_{i 2}$. Because Profile sets 1 and 2 have the first two attributes constant, we can write them as $\mathbf{X}_{i}=\left[\mathbf{1}_{2}, w_{i 1} \mathbf{1}_{2}, w_{i 2} \mathbf{1}_{2}, \mathbf{s}_{i 1}, \mathbf{s}_{i 2}\right]$, where $i=1,2$. The information matrix for each of the two profile sets then is

$$
\begin{aligned}
\mathbf{X}_{i}^{\prime} \mathbf{V}_{2}^{-1} \mathbf{X}_{i}= & {\left[\begin{array}{ccccc}
\mathbf{1}_{2}^{\prime} \mathbf{V}_{2}^{-1} \mathbf{1}_{2} & w_{i 1} \mathbf{1}_{2}^{\prime} \mathbf{V}_{2}^{-1} \mathbf{1}_{2} & w_{i 2} \mathbf{1}_{2}^{\prime} \mathbf{V}_{2}^{-1} \mathbf{1}_{2} & \mathbf{1}_{2}^{\prime} \mathbf{V}_{2}^{-1} \mathbf{s}_{i 1} & \mathbf{1}_{2}^{\prime} \mathbf{V}_{2}^{-1} \mathbf{s}_{i 2} \\
w_{i 1} \mathbf{1}_{2}^{\prime} \mathbf{V}_{2}^{-1} \mathbf{1}_{2} & w_{i 1}^{2} \mathbf{1}_{2}^{\prime} \mathbf{V}_{2}^{-1} \mathbf{1}_{2} & w_{i 1} w_{i 2} \mathbf{1}_{2}^{\prime} \mathbf{V}_{2}^{-1} \mathbf{1}_{2} & w_{i 1} \mathbf{1}_{2}^{\prime} \mathbf{V}_{2}^{-1} \mathbf{s}_{i 1} & w_{i 1} \mathbf{1}_{2}^{\prime} \mathbf{V}_{2}^{-1} \mathbf{s}_{i 2} \\
w_{i 2} \mathbf{1}_{2}^{\prime} \mathbf{V}_{2}^{-1} \mathbf{1}_{2} & w_{i 1} w_{i 2} \mathbf{1}_{2}^{\prime} \mathbf{V}_{2}^{-1} \mathbf{1}_{2} & w_{i 2}^{2} \mathbf{1}_{2}^{\prime} \mathbf{V}_{2}^{-1} \mathbf{1}_{2} & w_{i 2} \mathbf{1}_{2}^{\prime} \mathbf{V}_{2}^{-1} \mathbf{s}_{i 1} & w_{i 2} \mathbf{1}_{2}^{\prime} \mathbf{V}_{2}^{-1} \mathbf{s}_{i 2} \\
\mathbf{s}_{i 1}^{\prime} \mathbf{V}_{2}^{-1} \mathbf{1}_{2} & w_{i 1} \mathbf{s}_{i 1}^{\prime} \mathbf{V}_{2}^{-1} \mathbf{1}_{2} & w_{i 2} \mathbf{s}_{i 1}^{\prime} \mathbf{V}_{2}^{-1} \mathbf{1}_{2} & \mathbf{s}_{i 1}^{\prime} \mathbf{V}_{2}^{-1} \mathbf{s}_{i 1} & \mathbf{s}_{i 1}^{\prime} \mathbf{V}_{2}^{-1} \mathbf{s}_{i 2} \\
\mathbf{s}_{i 2}^{\prime} \mathbf{V}_{2}^{-1} \mathbf{1}_{2} & w_{i 1} \mathbf{s}_{i 2}^{\prime} \mathbf{V}_{2}^{-1} \mathbf{1}_{2} & w_{i 2} \mathbf{s}_{i 2}^{\prime} \mathbf{V}_{2}^{-1} \mathbf{1}_{2} & \mathbf{s}_{i 2}^{\prime} \mathbf{V}_{2}^{-1} \mathbf{s}_{i 1} & \mathbf{s}_{i 2}^{\prime} \mathbf{V}_{2}^{-1} \mathbf{s}_{i 2}
\end{array}\right] } \\
= & \sigma_{\boldsymbol{\varepsilon}}^{-2}\left[\begin{array}{ccccc}
2 c_{2} & 2 w_{i 1} c_{2} & 2 w_{i 2} c_{2} & c_{2} \mathbf{1}_{2}^{\prime} \mathbf{s}_{i 1} & c_{2} \mathbf{1}_{2}^{\prime} \mathbf{s}_{i 2} \\
2 w_{i 1} c_{2} & 2 c_{2} & 2 w_{i 1} w_{i 2} c_{2} & w_{i 1} c_{2} \mathbf{1}_{2}^{\prime} \mathbf{s}_{i 1} & w_{i 1} c_{2} \mathbf{1}_{2}^{\prime} \mathbf{s}_{i 2} \\
2 w_{i 2} c_{2} & 2 w_{i 1} w_{i 2} c_{2} & 2 c_{2} & w_{i 2} c_{2} \mathbf{1}_{2}^{\prime} \mathbf{s}_{i 1} & w_{i 2} c_{2} \mathbf{1}_{2}^{\prime} \mathbf{s}_{i 2} \\
c_{2} \mathbf{s}_{i 1}^{\prime} \mathbf{1}_{2} & w_{i 1} c_{2} \mathbf{s}_{i 1}^{\prime} \mathbf{1}_{2} & w_{i 2} c_{2} \mathbf{s}_{i 1}^{\prime} \mathbf{1}_{2} & \mathbf{s}_{i 1}^{\prime} \mathbf{s}_{i 1}-t_{11} & \mathbf{s}_{i 1}^{\prime} \mathbf{s}_{i 2}-t_{12} \\
c_{2} \mathbf{s}_{i 2}^{\prime} \mathbf{1}_{2} & w_{i 1} c_{2} \mathbf{s}_{i 2}^{\prime} \mathbf{1}_{2} & w_{i 2} c_{2} \mathbf{s}_{i 2}^{\prime} \mathbf{1}_{2} & \mathbf{s}_{i 2}^{\prime} \mathbf{s}_{i 1}-t_{21} & \mathbf{s}_{i 2}^{\prime} \mathbf{s}_{i 2}-t_{22}
\end{array}\right],
\end{aligned}
$$


where $c_{2}=(1-\rho) /(1+\rho), t_{11}=\frac{\rho}{1-\rho} c_{2}\left(\mathbf{s}_{i 1}^{\prime} \mathbf{1}_{2}\right)\left(\mathbf{1}_{2}^{\prime} \mathbf{s}_{i 1}\right), t_{12}=\frac{\rho}{1-\rho} c_{2}\left(\mathbf{s}_{i 1}^{\prime} \mathbf{1}_{2}\right)\left(\mathbf{1}_{2}^{\prime} \mathbf{s}_{i 2}\right)$, and $t_{21}$ and $t_{22}$ are obtained similarly to $t_{12}$ and $t_{11}$, respectively. Because the levels of the nonconstant attributes are balanced in the profile sets, $\mathbf{s}_{i 1}^{\prime} \mathbf{1}_{2}=\mathbf{1}_{2}^{\prime} \mathbf{s}_{i 1}=\mathbf{s}_{i 2}^{\prime} \mathbf{1}_{2}=\mathbf{1}_{2}^{\prime} \mathbf{s}_{i 2}=0$. As a result, filling out the entries of the formal information matrix (B.4) for profile set $i=1$ yields the information matrix

$$
\mathbf{X}_{1}^{\prime} \mathbf{V}_{2}^{-1} \mathbf{X}_{1}=\sigma_{\varepsilon}^{-2}\left[\begin{array}{ccccc}
+2 c_{2} & -2 c_{2} & -2 c_{2} & 0 & 0 \\
-2 c_{2} & +2 c_{2} & +2 c_{2} & 0 & 0 \\
-2 c_{2} & +2 c_{2} & +2 c_{2} & 0 & 0 \\
0 & 0 & 0 & +2 & +2 \\
0 & 0 & 0 & +2 & +2
\end{array}\right]
$$

and for profile set $i=2$ the information matrix is

$$
\mathbf{X}_{2}^{\prime} \mathbf{V}_{2}^{-1} \mathbf{X}_{2}=\sigma_{\varepsilon}^{-2}\left[\begin{array}{ccccc}
+2 c_{2} & -2 c_{2} & +2 c_{2} & 0 & 0 \\
-2 c_{2} & +2 c_{2} & -2 c_{2} & 0 & 0 \\
+2 c_{2} & -2 c_{2} & +2 c_{2} & 0 & 0 \\
0 & 0 & 0 & +2 & -2 \\
0 & 0 & 0 & -2 & +2
\end{array}\right]
$$

Summing these two matrices results in

$$
\sum_{i=1}^{2} \mathbf{X}_{i}^{\prime} \mathbf{V}_{2}^{-1} \mathbf{X}_{i}=\sigma_{\varepsilon}^{-2}\left[\begin{array}{ccccc}
+4 c_{2} & -4 c_{2} & 0 & 0 & 0 \\
-4 c_{2} & +4 c_{2} & 0 & 0 & 0 \\
0 & 0 & +4 c_{2} & 0 & 0 \\
0 & 0 & 0 & +4 & 0 \\
0 & 0 & 0 & 0 & +4
\end{array}\right]
$$

The diagonal elements for the part-worths in (B.7) reveal that, since $c_{2}<1$, less information is obtained on the constant attributes than on the non-constant attributes. If we had not included the constant attributes in the profile sets, the diagonal elements for the part-worths would have been all equal to four, the number of profiles in the two profile sets. Also, the two non-diagonal elements would have vanished.

The next pairs of profile sets in the design of Table A.12 have Attributes 1 and 4, 2 and 3 , and 3 and 4 as constant attributes. Hence, we denote Profile sets 3 and 4 as $\mathbf{X}_{i}=\left[\mathbf{1}_{2}, w_{i 1} \mathbf{1}_{2}, \mathbf{s}_{i 1}, \mathbf{s}_{i 2}, w_{i 2} \mathbf{1}_{2}\right]$, where $i=3,4$. We denote Profile sets 5 and 6 as $\mathbf{X}_{i}=\left[\mathbf{1}_{2}, \mathbf{s}_{i 1}, w_{i 1} \mathbf{1}_{2}, w_{i 2} \mathbf{1}_{2}, \mathbf{s}_{i 2}\right]$, where $i=5,6$ and we denote Profile sets 7 and 8 as $\mathbf{X}_{i}=$ $\left[\mathbf{1}_{2}, \mathbf{s}_{i 1}, \mathbf{s}_{i 2}, w_{i 1} \mathbf{1}_{2}, w_{i 2} \mathbf{1}_{2}\right]$, where $i=7,8$. By the same procedure as described above, the information matrices for each of these pairs are

$$
\sum_{i=3}^{4} \mathbf{X}_{i}^{\prime} \mathbf{V}_{2}^{-1} \mathbf{X}_{i}=\sigma_{\varepsilon}^{-2}\left[\begin{array}{ccccc}
+4 c_{2} & +4 c_{2} & 0 & 0 & 0 \\
+4 c_{2} & +4 c_{2} & 0 & 0 & 0 \\
0 & 0 & +4 & 0 & 0 \\
0 & 0 & 0 & +4 & 0 \\
0 & 0 & 0 & 0 & +4 c_{2}
\end{array}\right]
$$




$$
\begin{gathered}
\sum_{i=5}^{6} \mathbf{X}_{i}^{\prime} \mathbf{V}_{2}^{-1} \mathbf{X}_{i}=\sigma_{\varepsilon}^{-2}\left[\begin{array}{ccccc}
+4 c_{2} & 0 & 0 & -4 c_{2} & 0 \\
0 & +4 & 0 & 0 & 0 \\
0 & 0 & +4 c_{2} & 0 & 0 \\
-4 c_{2} & 0 & 0 & +4 c_{2} & 0 \\
0 & 0 & 0 & 0 & +4
\end{array}\right] \\
\sum_{i=7}^{8} \mathbf{X}_{i}^{\prime} \mathbf{V}_{2}^{-1} \mathbf{X}_{i}=\sigma_{\varepsilon}^{-2}\left[\begin{array}{ccccc}
+4 c_{2} & 0 & 0 & +4 c_{2} & 0 \\
0 & +4 & 0 & 0 & 0 \\
0 & 0 & +4 & 0 & 0 \\
+4 c_{2} & 0 & 0 & +4 c_{2} & 0 \\
0 & 0 & 0 & 0 & +4 c_{2}
\end{array}\right]
\end{gathered}
$$

Summing over the information matrices (B.7), (B.8), (B.9) and (B.10) for the four pairs of profile sets yields the total information matrix of the conjoint design:

$$
\begin{aligned}
\mathbf{X}^{\prime} \mathbf{V}^{-1} \mathbf{X} & =\sum_{i=1}^{8} \mathbf{X}_{i}^{\prime} \mathbf{V}_{2}^{-1} \mathbf{X}_{i} \\
& =\sigma_{\varepsilon}^{-2} \operatorname{diag}\left[\begin{array}{lllll}
16 c_{2} & 8 c_{2}+8 & 8 c_{2}+8 & 8 c_{2}+8 & 8 c_{2}+8
\end{array}\right]
\end{aligned}
$$

The amount of information on each of the part-worths is thus the same so that the conjoint design in Table A.12 is variance-balanced. The information component $8 c_{2}$ for each part-worth refers to the information on each attribute when it is constant. In the design each attribute is constant in 8 profiles. The other information component, 8 , for each part-worth points at the information on each attribute when it is non-constant. Each attribute is also non-constant in 8 profiles. If there were no constant attributes in the design, the diagonal elements for the part-worths would have been equal to 16, the number of profiles in the design. The information on the intercept always amounts to $16 c_{2}$.

An information matrix similar to the one in (B.11) can be derived for any of the optimal variance-balanced conjoint designs presented in this chapter. In the cases $m=4$ and $m=8$, the value for $c_{2}$ in the formulas is no longer appropriate and needs to be replaced by $c_{4}=(1-\rho) /(1+3 \rho)$ and $c_{8}=(1-\rho) /(1+7 \rho)$, respectively. Note that $c_{8}<c_{4}<c_{2}<1$. In general, to obtain the amount of information on each part-worth, two information components must be computed because of the partitioning in constant and non-constant attributes. The first information component contains $c_{m}$ multiplied by the number of profiles in which each attribute is constant. The second information component equals the number of profiles in which each attribute is non-constant. The amount of information on the intercept is obtained by multiplying the sample size by $c_{m}$. 


\section{References}

Abel, R. J. R. and Greig, M. (1996). BIBDs with small block size, in The CRC Handbook of Combinatorial Designs, Ch. I.2, Colbourn, C. J. and Dinitz, J. H., eds. Boca Raton (Florida): CRC Press, 41-47.

Brazier, J., Roberts, J. and Deverill, M. (2002). The estimation of a preference-based measure of health from the SF-36, Journal of Health Economics 21: 271-292.

Clatworthy, W. H. (1973). Tables of Two-Associate-Class Partially Balanced Designs, National Bureau of Standards (U.S.), Applied Mathematics Series 63.

Cochran, W. G. and Cox, G. M. (1957). Experimental Designs, New York: Wiley.

Cox, D. R. (1958). Planning of Experiments, New York: Wiley.

Danaher, P. J. (1997). Using conjoint analysis to determine the relative importance of service attributes measured in customer satisfaction surveys, Journal of Retailing 73: $235-260$.

Goos, P. (2002). The Optimal Design of Blocked and Split-Plot Experiments, New York: Springer.

Goos, P. (2006a). Optimal versus orthogonal and equivalent-estimation design of blocked and split-plot experiments, Statistica Neerlandica 60: 361-378.

Goos, P. (2006b). Optimal split-plot orthogonal main-effects plans, working paper, Department of Mathematics, Statistics and Actuarial Sciences, Faculty of Applied Economics, Universiteit Antwerpen, to appear.

Goos, P. and Vandebroek, M. (2001). Optimal split-plot designs, Journal of Quality Technology 33: 436-450.

Goos, P. and Vandebroek, M. (2004). Outperforming completely randomized designs, Journal of Quality Technology 36: 12-26.

Grasshoff, U., Grossmann, H., Holling, H. and Schwabe, R. (2004). Optimal designs for main effects in linear paired comparison models, Journal of Statistical Planning and Inference 126: 361-376.

Green, P. E. (1974). On the design of choice experiments involving multifactor alternatives, Journal of Consumer Research 1: 61-68. 
Grossmann, H., Holling, H., Brocke, M., Grasshoff, U. and Schwabe, R. (2005). On the empirical relevance of optimal designs for the measurement of preferences, in Applied Optimal Designs, Berger, M. and Wong, W.-K., eds. New York: Wiley, 45-65.

Grossmann, H., Holling, H. and Schwabe, R. (2002). Advances in optimum experimental design for conjoint analysis and discrete choice models, in Advances in Econometrics, Econometric Models in Marketing, Vol. 16, Franses, P. H. and Montgomery, A. L., eds. Amsterdam: JAI Press, 93-117.

Kessels, R., Goos, P. and Vandebroek, M. (2004). Optimal designs for rating-based conjoint experiments, working paper, Katholieke Universiteit Leuven, Belgium.

Mathon, R. and Rosa, A. (1996). 2- $(v, k, \lambda)$ designs of small order, in The CRC Handbook of Combinatorial Designs, Ch. I.1, Colbourn, C. J. and Dinitz, J. H., eds. Boca Raton (Florida): CRC Press, 3-41.

Pullman, M. E., Moore, W. L. and Wardell, D. G. (2002). A comparison of quality function deployment and conjoint analysis in new product design, Journal of Product Innovation Management 19: 354-364.

Schwabe, R., Grasshoff, U., Grossmann, H. and Holling, H. (2003). Optimal $2^{K}$ paired comparison designs for partial profiles, in PROBASTAT2002, Proceedings of the Fourth International Conference on Mathematical Statistics, Smolenice 2002, Tatra Mountains Mathematical Publications, Vol. 26, Stulajter, F. and Wimmer, G., eds., 79-86.

Severin, V. (2000). Comparing statistical efficiency and respondent efficiency in choice experiments, PhD Thesis, University of Sidney.

Shah, K. R. and Sinha, B. K. (1989). Theory of Optimal Designs, New York: SpringerVerlag.

Sinha, K. Website of partially balanced incomplete block designs: http://www.geocities. com/kishsinha2000/partiallybalanceddesigns.html.

Street, J., Bunch, D. S. and Moore, B. J. (2001). Optimal designs for $2^{k}$ paired comparison experiments, Communications in Statistics - Theory and Methods 30: 2149-2171.

Street, D. J. and Burgess, L. (2004). Optimal and near-optimal pairs for the estimation of effects in 2-level choice experiments, Journal of Statistical Planning and Inference 118: $185-199$. 
Street, D. J. and Street, A. P. (1996). Partially balanced incomplete block designs, in The CRC Handbook of Combinatorial Designs, Colbourn, C. J. and Dinitz, J. H., Ch. IV.35, eds. Boca Raton (Florida): CRC Press, 419-423.

Wu, C. F. J. and Hamada, M. (2000). Experiments: Planning, Analysis, and Parameter Design Optimization, New York: Wiley. 